\title{
1 Synergistic Coding of Human Odorants in the Mosquito Brain
}

3 Shruti Shankar ${ }^{1}$, Genevieve M. Tauxe ${ }^{1}$, Emma D. Spikol ${ }^{2}$, Ming Li ${ }^{3}$, Omar S. Akbari ${ }^{3}$, Diego

4 Giraldo ${ }^{1}$, Conor J. McMeniman 1, 2, *

$6{ }^{1}$ W. Harry Feinstone Department of Molecular Microbiology and Immunology, Johns Hopkins

7 Malaria Research Institute, Johns Hopkins Bloomberg School of Public Health, Johns Hopkins

8 University, Baltimore, MD 21205, USA.

102 The Solomon H. Snyder Department of Neuroscience, Johns Hopkins University School of 11 Medicine, Baltimore, MD 21205, USA.

${ }^{3}$ Section of Cell and Developmental Biology, University of California, San Diego, La Jolla, CA, 14 92093, USA.

${ }^{*}$ Corresponding author

Email: cmcmeni1@jhu.edu

\section{Abstract:}

The yellow fever mosquito Aedes aegypti employs olfaction to locate humans. We applied CRISPR-Cas9 genome engineering and neural activity mapping to define the molecular and cellular logic of how the mosquito brain is wired to detect human odorants. We determined that the breath volatile carbon dioxide $\left(\mathrm{CO}_{2}\right)$ is detected by the largest unit of olfactory coding in the primary olfactory processing center of the mosquito brain, the antennal lobe. Synergistically, $\mathrm{CO}_{2}$ detection gates synaptic transmission from defined populations of olfactory sensory neurons, innervating unique antennal lobe regions tuned to the human sweat odorant L-(+)-lactic acid. Our data suggests that simultaneous detection of signature human volatiles rapidly disinhibits a multimodal olfactory network for hunting humans in the mosquito brain. 


\section{Main Text:}

Blood-thirsty female $A$. aegypti mosquitoes detect and navigate towards a plethora of physical and chemosensory cues emitted by the human body (1-4). Of these cues, human scent is a powerful mosquito attractant, comprising of a complex bouquet of hundreds of volatile chemicals derived from sweat, breath and the human skin microbiome (5). Despite recent advances in our understanding of mosquito chemoreception at the periphery $(2,6-13)$, central mechanisms involved in detection and integration of human body odorants by this prolific disease vector are largely unknown (2). This highlights the critical need for novel approaches to illuminate olfactory circuits underlying the epidemiologically important process of $A$. aegypti attraction to human body odor.

The olfactory system of $A$. aegypti consists of three major olfactory appendages including the antennae, maxillary palps and labella of the proboscis. Lining these organs are various morphological classes of porous sensilla (14-16) that house the dendritic processes of typically 2-3 olfactory sensory neurons (OSNs) that detect diverse structural classes of volatile odorants. The axonal processes of OSNs project to the primary olfactory processing brain center known as the antennal lobe (17). In related insects such as Drosophila, olfactory information is locally processed and encoded in the antennal lobe via the action of excitatory and inhibitory local neurons (18-20), before being sent by projection neurons to higher order brain centers involved in orchestrating innate and learned olfactory behaviors (21-23).

Large chemoreceptor gene families implicated in detection of various components of human scent and other ethologically relevant odorants are encoded in the A. aegypti genome (24). The Odorant Receptor $(O R)$ chemoreceptor family, typically tuned to aldehydes, short-chain alcohols and ketones, likely mediates anthropophilic host preference in $A$. aegypti $(8,12)$. In a complementary

61 fashion, chemoreceptors from the lonotropic Receptor (IR) family that are responsive to carboxylic 62 acids and amines (25), and certain Gustatory Receptors $(G R)$ family members that detect the 63 volatile gas carbon dioxide $\left(\mathrm{CO}_{2}\right)(2)$, drive synergistic behavioral taxis of female mosquitoes 64 towards human scent $(26,27)$. For instance, L-(+)-lactic Acid, a predominant chemical fraction of 65 human sweat, is alone unattractive to $A$. aegypti, but potently synergizes with $\mathrm{CO}_{2}$ to elicit 66 olfactory attraction when these two stimuli are combined together $(28,29)$. Functionality of the $I R$ 67 co-receptor IR8a that putatively forms multimeric IR complexes tuned to L-(+)-lactic acid and related acidic volatiles (25), as well as the $\mathrm{Gr} 1 / 2 / 3 \mathrm{CO}_{2}$ receptor complex (2), are together required 
for this olfactory synergism. However, the mechanistic basis of how these and other human volatiles are integrated by the $A$. aegypti antennal lobe to yield attractive behavioral synergy is currently unclear.

To identify antennal lobe circuits mediating synergy between $\mathrm{CO}_{2}$ and L-(+)-lactic acid in the mosquito brain, we first developed an updated in vitro neuroanatomical atlas of the antennal lobe from the LVPib12 A. aegypti strain, demonstrating that this olfactory brain center contains $\sim 80$ units of glomerular synaptic connectivity (17). In Drosophila, the axonal processes of OSNs expressing unique complements of chemoreceptors project from the peripheral sensory appendages such as the antenna and maxillary palp to spatially defined glomeruli within the antennal lobe $(30,31)$. To facilitate in-depth neuroanatomical studies and genetic access to the A. aegypti antennal lobe, we first applied CRISPR-Cas9 genome engineering (32) and Mos1mariner transposition (33) to integrate components of the QF2/QUAS system (34) for binary expression of reporter transgenes in defined subsets of $A$. aegypti OSNs projecting to this brain region.

To generate transgenic $A$. aegypti chemoreceptor-QF2 driver lines, we used CRISPR-Cas9 mediated homologous recombination to insert a T2A-QF2 in-frame fusion cassette (35) into the coding exons of three major olfactory co-receptor genes: Odorant Receptor co-receptor (orco), Ionotropic Receptor co-receptor IR8a, and the $\mathrm{CO}_{2}$ receptor complex subunit Gr1 (Fig. 1A-C). Using this strategy, QF2 was integrated in-frame into Exon 3 of each target gene, placing expression of this transcription factor under control of endogenous regulatory elements for each locus. These driver lines also included a visible $3 \times P 3-D s R e d 2$ eye marker to facilitate identification of transgenic individuals. orco ${ }^{Q F 2 R e d}$ and IR8a ${ }^{Q F 2 R e d}$ cassettes inserted in-frame as expected via ends-out recombination events, whereas Gr1 ${ }^{\text {QF2Red }}$ inserted in-frame yet also incorporated a duplicated copy of the plasmid backbone downstream of the T2A-QF2 in-frame fusion via an ends-in recombination event.

We crossed each driver line with a QUAS-mCD8::GFP responder strain that we generated by Mos1 mariner transposition and demonstrated that we successfully labeled OSNs with membrane-tethered green fluorescent protein (GFP). Confocal analyses of female peripheral sensory appendages revealed strong GFP expression in OSN dendrites and cell bodies on the antenna, maxillary palp and labella of the proboscis of orco ${ }^{\text {QF2Red }}>15 X Q U A S-m C D 8:: G F P$ individuals (Fig. 1a and Fig. S1 a-e); as well as OSN labeling of the antennal flagellum of 
IR8a ${ }^{\text {QF2Red }}>15 X Q U A S-m C D 8:: G F P$ (Fig. 1b) individuals, and maxillary palp tissue of

104 Gr1 ${ }^{\text {QF2Red }}>15 X Q U A S-m C D 8:: G F P$ mosquitoes (Fig. 1c).

Expression patterns from QF2 knock-ins were consistent with a previous LVPib12 strain neurotranscriptome analysis (36) that revealed broad orco expression across olfactory tissues, with IR8a and Gr1 expression confined to antennal and maxillary palp tissue, respectively. Dendrites of orco $(+)$ neurons on the mosquito antenna were localized to hair-like trichoid sensilla (Fig. 1a), whereas dendrites of IR8a (+) neurons were confined to grooved-peg sensilla on the antenna, and Gr1 (+) neurons were found in capitate peg sensilla on the maxillary palp (Fig. 1b and c). These latter two classes of sensilla are the locations for OSN-based detection of L-(+)lactic acid $(37,38)$ and $\mathrm{CO}_{2}(2,39,40)$, respectively, grossly validating the neuroanatomical specificity of our transgenic labeling approach.

To examine central projection patterns of OSNs expressing the $\mathrm{CO}_{2}$ receptor complex subunit antibody directed against the pre-synaptic protein Bruchpilot (BRP) (41) to demarcate glomerular boundaries of neuropils in the antennal lobe, and anti-GFP antibody to amplify mCD8::GFP signal.

121 Surprisingly, immunohistochemistry with $Q F 2^{\text {Red }}$ genotypes revealed spurious red and green

122 fluorescence throughout the central brain (Fig. S2 a-c), particularly in glia, including in fixed brains 123 not subjected to anti-GFP staining, suggesting potential interference in the expected QF2/QUAS 124 transactivation pattern at these loci. As all of our T2A-QF2 insertions included a downstream 125 fluorescent marker cassette containing the $3 x P 3$ synthetic promoter (42), which is a multimerized 126 binding site for the paired-box transcription factor Pax6 involved in glial and neuronal development $127(43,44)$, we suspected that the source of the this aberrant expression pattern may be due to 128 promiscuous $3 \times P 3$ enhancer activity operating at these genomic loci.

To abrogate this effect, we developed a strategy to excise floxed $3 x P 3$ fluorescent marker 131 cassettes from our $Q F 2^{R e d}$ strains via crossing these genotypes to a germline Cre recombinase 132 strain (exu-Cre) that we engineered. Using this approach, we successfully generated marker-free 133 driver strains (orco ${ }^{Q F 2}, I R 8 a^{Q F 2}$ and $G r 1^{Q F 2}$ ) which were devoid of all $3 x P 3$ fluorescent markers 134 and any apparent background fluorescence in the central brain, clearly driving reporter expression 135 in OSN axonal processes innervating the antennal lobe (Fig. 1, d-f and Fig. S2 d-i). Consistent 136 with peripheral expression patterns, in marker-free QF2>mCD8:GFP composite genotypes, orco 
(+) OSNs were observed to innervate the largest number of glomeruli (60/78 total) across several spatial regions of the antennal lobe, whereas IR8a (+) neurons (15/78 total) and Gr1 (+) neurons (1/78 total) innervate sparser subsets of glomeruli in posterolateral and mediodorsal antennal lobe regions, respectively (Fig. $2 a)$.

Using a systematic reference key for $A$. aegypti antennal lobe nomenclature (17), we then determined that a subset of 6 orco/IR8a glomeruli had putative co-labeling, indicative of coexpression of these two genes, based on their overlapping positions and assigned name relative to defined antennal lobe landmarks. We also found 8/78 total glomeruli in the ventral region of the antennal lobe that were not labeled by any of these chemoreceptor driver lines (Fig. 2a), and we suggest these glomeruli may receive innervations from OSNs expressing other chemoreceptors such as those complexed with IR co-receptors IR25a and IR76b, which are known to project to the antennal lobe in Drosophila (45). Additionally, we visualized orco (+) neurons innervating the taste center of the insect brain known as the suboesophageal zone (SEZ) (Fig. S3), consistent with the projection pattern of orco (+) OSNs in the African malaria mosquito Anopheles gambiae (46). 2D and 3D mapping of antennal lobes from replicate brain samples (Fig 2, Fig. S4-S7), revealed the same complement of glomeruli was consistently labeled across orco, IR8a and Gr1 driver strains. Volumetric analysis of $A$. aegypti antennal lobe glomeruli from each of these clusters of chemoreceptor innervation further revealed that the Gr1 (+) glomerulus MD1 is the largest glomerulus in the antennal lobe (Fig. 2b). This observation may reflect the critical importance of $\mathrm{CO}_{2}$ to multiple facets of $A$. aegypti host-seeking behavior $(1-4,26,27)$.

Markedly increased glomerular subdivision of the $A$. aegypti antennal lobe relative to Drosophila (17) makes assigning odor-evoked neurophysiological responses to particular glomeruli at highspatial resolution a daunting challenge. To leverage the detailed receptor-to-glomerulus antennal 162 lobe reference maps we generated for orco (+), IR8a (+) and Gr1 (+) OSNs (Fig 2 and Fig. S4163 S7), we next applied functional imaging assays with calcium modulated photoactivatable 164 ratiometric indicator (CaMPARI2) (47) to assess neural correlates of olfactory synergy between $165 \mathrm{CO}_{2}$ and L-(+)-lactic acid. This genetically encoded, ratiometric calcium indicator photoconverts 166 from green to red in the presence of $405 \mathrm{~nm}$ light and high levels of calcium $(47,48)$, providing a 167 permanent readout of neural activity during odorant stimulation. CaMPARI2 is further amenable 168 to post-hoc staining methods for spatial registration of neural activity (47). We localized 169 photoconversion signal from this fluorescent indicator to specific antennal lobe glomeruli using brain co-staining with fluorophore-conjugated phalloidin toxin to mark cytoskeletal F-actin 
171 filaments in OSN axonal processes (49), with reference to phalloidin-stained antennal lobe maps

172 that we generated (Fig. S8).

173

174 To initially trial the efficacy of CaMPARI2 at recording odor-evoked activity from $A$. aegypti 175 antennal lobe glomeruli, we calculated photoconversion ratios in the axon terminals of Gr1 (+) 176 OSNs innervating the MD1 glomerulus in response to stimulation with $\mathrm{CO}_{2}$. To do this, we first 177 generated composite imaging strains that express CaMPAR/2 driven by a cumulative 30 copies 178 of the $Q$ upstream activation sequence in $\mathrm{Gr} 1$ (+) OSNs (denoted here as Gr1 ${ }^{\text {QF2 }}>30 X Q U A S$ 179 CaMPARI2, Table S10). The olfactory appendages of live head-tethered mosquito preparations, 180 with surgically exposed antennal lobes, were then subjected to a standard CaMPARI stimulus 181 duty cycle (48) consisting of simultaneous pulses of $405 \mathrm{~nm}$ photoconversion light through a highnumerical aperture water immersion objective and $1 \% \mathrm{CO}_{2}$ delivered by a custom olfactometer. After each photoconversion regime, brains were immediately dissected from each mosquito, costained with fluorophore-conjugated toxin phalloidin to demarcate glomerular boundaries with confocal imaging, and green to red CaMPARI2 photoconversion ratios in MD1 were calculated to query whether the axonal terminals of $\mathrm{Gr}_{1}(+)$ OSNs projecting to this glomerulus responded to the stimulus. Encouragingly, MD1 exhibited a significantly higher rate of CaMPARI2 photoconversion in $\mathrm{CO}_{2}$-stimulated mosquitoes versus those that were stimulated with synthetic air (Fig. 3 a-c), further validating the ability of $405 \mathrm{~nm}$ light to penetrate mosquito brain tissue and photoconvert glomeruli such as MD1 positioned deep below the antennal lobe surface (17).

Having demonstrated CAMPARI2 photoconversion was a viable approach for activity-dependent neural labeling in OSN axon terminals innervating the antennal lobe, we then went on to test whether synergy between the sweat odorant L-(+)-lactic acid and $\mathrm{CO}_{2}$ could be detected in specific IR8a (+) glomeruli, given that IR8a (25) and the $\mathrm{CO}_{2}$ receptor pathways (2) are both required for synergistic attraction of $A$. aegypti to these two odorants. To do this, we mapped CaMPARI2 activity in 12 IR8a (+) glomeruli in replicate brain samples derived from IR8a ${ }^{Q F 2}>$ 30XQUAS-CaMPAR/2 mosquitoes that were stimulated with unitary and binary combinations of L-(+)-lactic acid and CO2. Surprisingly, we determined that application of L-(+)-lactic acid alone

200 to olfactory appendages of live imaging mosquitoes did not yield higher CaMPARI2 201 photoconversion ratios than $\mathrm{CO} 2$ or synthetic air controls in IR8a (+) glomeruli (Fig. 4 b, C, d). In 202 contrast, we observed a dramatic increase in CaMPARI2 photoconversion ratios when $\mathrm{CO}_{2}$ was 203 coapplied with L-(+)-lactic acid to mosquitoes (Fig. 4 a). In particular, two out of twelve IR8a (+) 204 glomeruli, denoted PL5 and PL6, exhibited synergistic and highly significant differences in mean 
CaMPARI2 photoconversion values when co-stimulated with $\mathrm{CO}_{2}$ and $\mathrm{L}-(+)$-lactic acid relative to L-(+)-lactic acid or $\mathrm{CO}_{2}$ alone and synthetic air controls (Fig. 4 e and f, Fig. S9 and S10). We interpret this as evidence that presynaptic calcium levels are significantly elevated in IR8a (+) OSN axon terminals innervating PL5 and PL6 in response to co-stimulation with $\mathrm{CO}_{2}$ and L-(+)lactic acid.

This study lays the critical foundation towards defining how constituents of human scent and other chemosensory stimuli are encoded in the mosquito brain. Our data are highly suggestive of the existence of a circuit-based mechanism for olfactory synergism between the human sweat odorant L-(+)-lactic acid and breath volatile $\mathrm{CO}_{2}$ at first olfactory synapse in the antennal lobe. We observed that axon terminals of IR8a (+) neurons had consistently low CaMPARI2 photoconversion signals in response to stimulation with L-(+)-lactic acid or CO2 alone, that were not significantly different from baseline values observed with clean air. Synergistically, presynaptic calcium levels in axon terminals of $I R 8 a(+)$ neurons were dramatically elevated upon co-stimulation of mosquitoes with L-(+)-lactic acid with $\mathrm{CO}_{2}$, as reported by enhanced CaMPARI2 photoconversion in PL5 and PL6 glomeruli.

Previous extracellular recordings on the A. aegypti antenna demonstrated no changes in odorevoked activity from L-(+)-lactic acid-sensitive OSNs in response to stimulation with this carboxylic acid with $\mathrm{CO}_{2}$ (38) indicating synergism likely does not occur though peripheral mechanisms $(50,51)$ during ligand detection by OSN dendrites. Rather, we speculate given the silent nature of IR8a (+) glomerular responses towards unitary blends of either ligand, that this binary synergy may occur via disinhibitory local circuitry (52) operating between the $\mathrm{CO}_{2}$-sensitive glomerulus MD1 and axon terminals of lactic acid-sensitive IR8a (+ ) glomeruli such as PL5 and PL6 in the antennal lobe.

Rapid central feedback between antennal lobe glomeruli to yield disinhibition of OSN axon

232 terminals may therefore represent a simple, yet flexible circuit for this prolific disease vector to

233 faithfully identify signature combinations of human odorants and improve the fidelity of their hunt

234 for humans. Given that multiple human odorants in combination likely lie at the heart of mosquito 235 lust for human scent $(53,54)$, further dissection of synergistic coding operational in the mosquito antennal lobe may reveal key human volatiles and mosquito chemosensory circuitry that can be targeted to combat mosquito-borne diseases such as dengue, Zika and malaria. 


\section{References:}

1. R. A. Corfas, L. B. Vosshall, The cation channel TRPA1 tunes mosquito thermotaxis to host temperatures. Elife 4, (2015).

2. C. J. McMeniman, R. A. Corfas, B. J. Matthews, S. A. Ritchie, L. B. Vosshall, Multimodal integration of carbon dioxide and other sensory cues drives mosquito attraction to humans. Cell 156, 1060-1071 (2014).

3. F. van Breugel, J. Riffell, A. Fairhall, M. H. Dickinson, Mosquitoes Use Vision to Associate Odor Plumes with Thermal Targets. Curr Biol 25, 2123-2129 (2015).

4. C. Vinauger et al., Visual-Olfactory Integration in the Human Disease Vector Mosquito Aedes aegypti. Curr Biol 29, 2509-2516.e2505 (2019).

5. L. Dormont, J. M. Bessière, A. Cohuet, Human skin volatiles: a review. J Chem Ecol 39, 569-578 (2013).

6. A. F. Carey, G. Wang, C. Y. Su, L. J. Zwiebel, J. R. Carlson, Odorant reception in the malaria mosquito Anopheles gambiae. Nature 464, 66-71 (2010).

7. Z. Chen, F. Liu, N. Liu, Human Odour Coding in the Yellow Fever Mosquito, Aedes aegypti. Sci Rep 9, 13336 (2019).

8. M. DeGennaro et al., orco mutant mosquitoes lose strong preference for humans and are not repelled by volatile DEET. Nature 498, 487-491 (2013).

9. E. A. Hallem, A. Nicole Fox, L. J. Zwiebel, J. R. Carlson, Olfaction: mosquito receptor for human-sweat odorant. Nature 427, 212-213 (2004).

10. W. D. Jones, P. Cayirlioglu, I. G. Kadow, L. B. Vosshall, Two chemosensory receptors together mediate carbon dioxide detection in Drosophila. Nature 445, 86-90 (2007).

11. T. Lu et al., Odor coding in the maxillary palp of the malaria vector mosquito Anopheles gambiae. Curr Biol 17, 1533-1544 (2007).

12. C. S. McBride et al., Evolution of mosquito preference for humans linked to an odorant receptor. Nature 515, 222-227 (2014).

13. G. Wang, A. F. Carey, J. R. Carlson, L. J. Zwiebel, Molecular basis of odor coding in the malaria vector mosquito Anopheles gambiae. Proc Natl Acad Sci U S A 107, 4418-4423 (2010).

14. S. B. Mclver, Structure of sensilla trichodea of female Aedes aegypti with comments on innervation of antennal sensilla. Journal of Insect Physiology 24, 383-390 (1978).

15. S. B. Mclver, Fine structure of pegs on the palps of female culicine mosquitoes. Can J Zool 50, 571-576 (1972).

16. S. B. Mclver, Fine structure of antennal sensilla coeloconica of culicine mosquitoes. Tissue Cell 5, 105-112 (1973).

17. S. Shankar, C. J. McMeniman, An updated antennal lobe atlas for the yellow fever mosquito Aedes aegypti. PLoS Negl Trop Dis 14, e0008729 (2020).

18. Y. H. Chou et al., Diversity and wiring variability of olfactory local interneurons in the Drosophila antennal lobe. Nat Neurosci 13, 439-449 (2010).

19. E. J. Hong, R. I. Wilson, Simultaneous encoding of odors by channels with diverse sensitivity to inhibition. Neuron 85, 573-589 (2015).

20. E. Yaksi, R. I. Wilson, Electrical coupling between olfactory glomeruli. Neuron 67, 10341047 (2010).

21. S. J. Caron, V. Ruta, L. F. Abbott, R. Axel, Random convergence of olfactory inputs in the Drosophila mushroom body. Nature 497, 113-117 (2013).

22. M. J. Dolan et al., Neurogenetic dissection of the Drosophila lateral horn reveals major outputs, diverse behavioural functions, and interactions with the mushroom body. Elife $\mathbf{8}$, (2019). 
23. V. Grabe et al., Elucidating the Neuronal Architecture of Olfactory Glomeruli in the Drosophila Antennal Lobe. Cell Rep 16, 3401-3413 (2016).

24. B. J. Matthews et al., Improved reference genome of Aedes aegypti informs arbovirus vector control. Nature 563, 501-507 (2018).

25. J. I. Raji et al., Aedes aegypti Mosquitoes Detect Acidic Volatiles Found in Human Odor Using the IR8a Pathway. Curr Biol 29, 1253-1262.e1257 (2019).

26. T. Dekker, R. T. Cardé, Moment-to-moment flight manoeuvres of the female yellow fever mosquito (Aedes aegypti L.) in response to plumes of carbon dioxide and human skin odour. J Exp Biol 214, 3480-3494 (2011).

27. T. Dekker, M. Geier, R. T. Cardé, Carbon dioxide instantly sensitizes female yellow fever mosquitoes to human skin odours. J Exp Biol 208, 2963-2972 (2005).

28. F. Acree, Jr., R. B. Turner, H. K. Gouck, M. Beroza, N. Smith, L-Lactic acid: a mosquito attractant isolated from humans. Science 161, 1346-1347 (1968).

29. A. E. Eiras, P. C. Jepson, Host location by Aedes aegypti (Diptera: Culicidae): a wind tunnel study of chemical cues. Bulletin of Entomological Research 81, 151-160 (1991).

30. A. Couto, M. Alenius, B. J. Dickson, Molecular, anatomical, and functional organization of the Drosophila olfactory system. Curr Biol 15, 1535-1547 (2005).

31. L. B. Vosshall, A. M. Wong, R. Axel, An olfactory sensory map in the fly brain. Cell 102, 147-159 (2000).

32. K. E. Kistler, L. B. Vosshall, B. J. Matthews, Genome engineering with CRISPR-Cas9 in the mosquito Aedes aegypti. Cell Rep 11, 51-60 (2015).

33. C. J. Coates, N. Jasinskiene, L. Miyashiro, A. A. James, Mariner transposition and transformation of the yellow fever mosquito, Aedes aegypti. Proc Natl Acad Sci U S A 95, 3748-3751 (1998).

34. O. Riabinina et al., Improved and expanded Q-system reagents for genetic manipulations. Nat Methods 12, 219-222, 215 p following 222 (2015).

35. F. Diao, B. H. White, A novel approach for directing transgene expression in Drosophila: T2A-Gal4 in-frame fusion. Genetics 190, 1139-1144 (2012).

36. B. J. Matthews, C. S. McBride, M. DeGennaro, O. Despo, L. B. Vosshall, The neurotranscriptome of the Aedes aegypti mosquito. BMC Genomics 17, 32 (2016).

37. E. E. Davis, Structure-response relationship of the lactic acid-excited neurones in the antennal grooved-peg sensilla of the mosquito Aedes aegypti. Journal of Insect Physiology 34, 443-449 (1988).

38. E. E. Davis, P. G. Sokolove, Lactic acid-sensitive receptors on the antennae of the mosquito,Aedes aegypti. Journal of comparative physiology 105, 43-54 (1976).

39. A. J. Grant, B. E. Wigton, J. G. Aghajanian, R. J. O'Connell, Electrophysiological responses of receptor neurons in mosquito maxillary palp sensilla to carbon dioxide. $J$ Comp Physiol A 177, 389-396 (1995).

40. F. E. Kellogg, Water vapour and carbon dioxide receptors in Aedes aegypti. J Insect Physiol 16, 99-108 (1970).

41. A. Hofbauer et al., The Wuerzburg hybridoma library against Drosophila brain. $J$ Neurogenet 23, 78-91 (2009).

42. A. J. Berghammer, M. Klingler, E. A. Wimmer, A universal marker for transgenic insects. Nature 402, 370-371 (1999).

43. R. Quiring, U. Walldorf, U. Kloter, W. J. Gehring, Homology of the eyeless gene of Drosophila to the Small eye gene in mice and Aniridia in humans. Science 265, 785-789 (1994).

44. T. Suzuki, R. Takayama, M. Sato, eyeless/Pax6 controls the production of glial cells in the visual center of Drosophila melanogaster. Dev Biol 409, 343-353 (2016).

45. A. F. Silbering et al., Complementary function and integrated wiring of the evolutionarily distinct Drosophila olfactory subsystems. J Neurosci 31, 13357-13375 (2011). 
46. O. Riabinina et al., Organization of olfactory centres in the malaria mosquito Anopheles

47. B. Moeyaert et al., Improved methods for marking active neuron populations. Nat Commun 9, 4440 (2018).

48. B. F. Fosque et al., Neural circuits. Labeling of active neural circuits in vivo with designed calcium integrators. Science 347, 755-760 (2015).

49. W. Rössler, J. Kuduz, F. W. Schürmann, D. Schild, Aggregation of f-actin in olfactory glomeruli: a common feature of glomeruli across phyla. Chem Senses 27, 803-810 (2002).

50. C. Y. Su, K. Menuz, J. Reisert, J. R. Carlson, Non-synaptic inhibition between grouped neurons in an olfactory circuit. Nature 492, 66-71 (2012).

51. L. Xu et al., Widespread receptor-driven modulation in peripheral olfactory coding. Science 368, eaaz5390 (2020).

52. W. Zhao et al., A disinhibitory mechanism biases Drosophila innate light preference. Nat Commun 10, 124 (2019).

53. U. R. Bernier et al., Synergistic attraction of Aedes aegypti (L.) to binary blends of Llactic acid and acetone, dichloromethane, or dimethyl disulfide. J Med Entomol 40, 653656 (2003).

54. R. C. Smallegange, Y. T. Qiu, J. J. van Loon, W. Takken, Synergism between ammonia, lactic acid and carboxylic acids as kairomones in the host-seeking behaviour of the malaria mosquito Anopheles gambiae sensu stricto (Diptera: Culicidae). Chem Senses 30, 145-152 (2005).

55. V. Nene et al., Genome sequence of Aedes aegypti, a major arbovirus vector. Science 316, 1718-1723 (2007).

56. M. Li et al., Germline Cas9 expression yields highly efficient genome engineering in a major worldwide disease vector, Aedes aegypti. Proc Natl Acad Sci U S A 114, E10540e10549 (2017).

57. C. J. Potter, L. Luo, Splinkerette PCR for mapping transposable elements in Drosophila. PLoS One 5, e10168 (2010).

58. J. M. Richardson, S. D. Colloms, D. J. Finnegan, M. D. Walkinshaw, Molecular architecture of the Mos1 paired-end complex: the structural basis of DNA transposition in a eukaryote. Cell 138, 1096-1108 (2009).

59. K. W. Beyenbach, R. Masia, Membrane conductances of principal cells in Malpighian tubules of Aedes aegypti. J Insect Physiol 48, 375-386 (2002). 


\section{Acknowledgements:}

We thank N. Kizito, B. Natarajan, H. Rosado, M. Gebhardt, V. Balta and B. Burgunder for expert technical assistance; R. Harrell (UM-ITF) for mosquito embryonic microinjection services; S. Seo and A. Hammond for help with transgene mapping, C. Potter and E. Schreiter for constructs and technical advice; and C. Huang, M. Schnitzer, B. Ferris, G. Maimon, C. Dan and V. Jayaraman for guidance on surgical preparations. This research was supported by funding from the National Institutes of Health NIAID (R21 Al139358-01), USAID (AID-OAA-F-16-00061) and Centers for Disease Control and Prevention (200-2017-93143) to C.J.M; and funding to G.M.T. as a postdoctoral fellow on The Molecular And Cellular Basis Of Infectious Diseases (MCBID) Program (T32A1007417) from the NIH. O.S.A and M.L. were supported in part by a DARPA Safe Genes Program Grant (HR0011-17-2-0047) and a DARPA ReVector program grant (HR0011-20-2-0030) awarded to O.S.A. The views, opinions and/or findings expressed should not be interpreted as representing the official views or policies of the Department of Defense or the U.S. Government. Microscopy infrastructure at Johns Hopkins School of Medicine Microscope Core Facility used in this research was supported by the National Institutes of Health NCRR (S100D016374 and S10OD023548). The mosquito template in Figure 1 was created for us by Biorender.com. We thank Terry Shelley at the JHU Center for Neuroscience Research Machine Shop for fabrication services supported by NINDS Center grant (NS050274). We further acknowledge generous support to C.J.M. from Johns Hopkins Malaria Research Institute (JHMRI) and Bloomberg Philanthropies. S.S. and G.M.T. were supported by JHMRI Postdoctoral Fellowships.

\section{Contributions:}

S.S. and C.J.M conceived the experimental design. M.L. and O.S.A. generated and provided the exu-Cas9 strain for use. G.M.T., C.J.M. and S.S. together engineered constructs for transgenesis and the custom olfactometer for odorant delivery. C.J.M. and G.M.T. screened, genotyped and maintained transgenic lines. E.D.S. performed confocal analyses of IR8a peripheral expression patterns. S.S. performed all other microscopy, immunohistochemistry, antennal lobe reconstructions, glomerular mapping and CaMPARI2 imaging experiments. D.G. and S.S. analyzed the data. S.S. and C.J.M. drafted the manuscript.

\section{Competing interests:}

The authors declare no competing interests.

Materials \& Correspondence: Correspondence to Conor J. McMeniman 
a

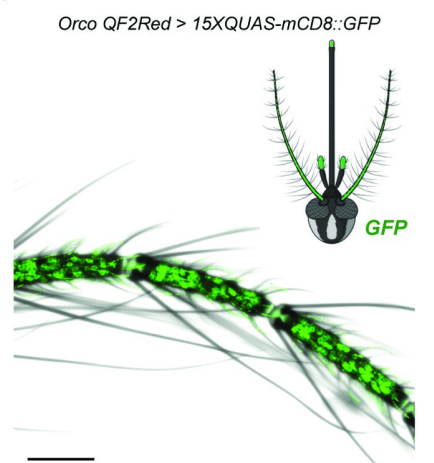

d

Orco $Q F 2>30 X Q U A S-m C D 8:: G F P$

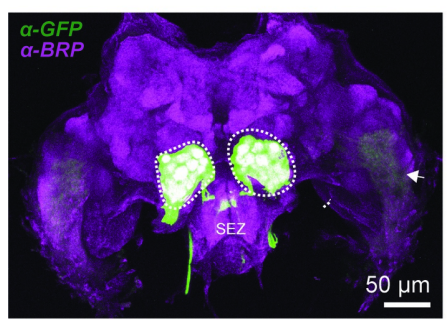

b

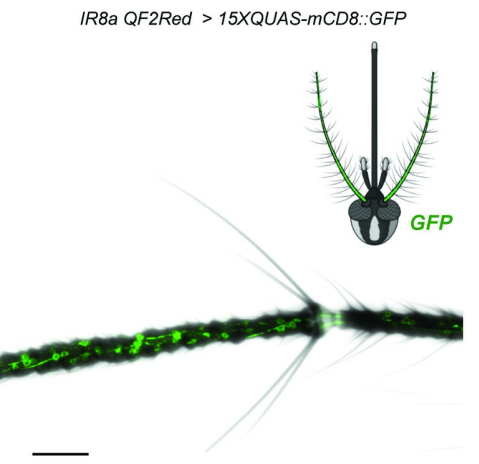

e Gr1 QF2Red > 15XQUAS-mCD8::GFP

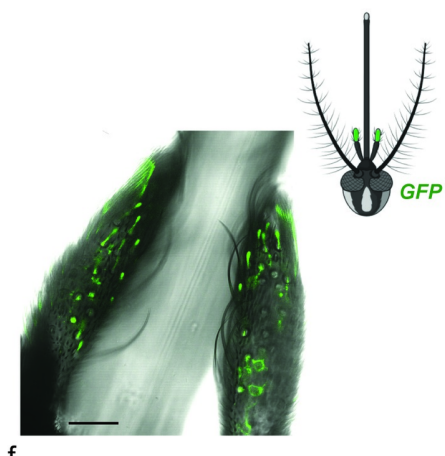

Gr1 QF2 > 30XQUAS-mCD8::GFP
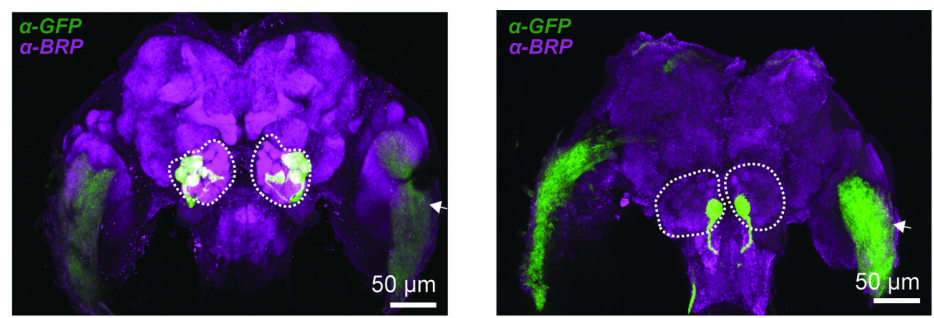

Figure 1. OSNs expressing divergent chemoreceptor gene families project centrally to defined regions of the

411 Aedes aegypti antennal lobe. To study the expression patterns of orco, IR8a and Gr1 in the nervous system of female

412 A. aegypti, each of the three chemoreceptor QF2 driver lines we generated was crossed to a QUAS mCD8::GFP reporter line and peripheral and central innervation patterns were imaged using confocal microscopy. (a) Densely packed cell bodies of orco (+) OSNs were observed in all 13 antennal flagellomeres, with dendrites localized to trichoid sensilla along the antennal surface, as well as the sensilla on the labella of the proboscis and maxillary palps (see Figure S1); (b) IR8a (+) OSNs were housed on all antennal flagellomeres in grooved peg sensilla; while (c) Gr1 (+) OSNs were visualized exclusively in the club-shaped capitate peg sensilla on the ventral surface of the maxillary palps. Projections to the mosquito brain: (d) orco $(+)$ neurons innervate the entire anterior region of the antennal lobe as well as the suboesophageal zone (SEZ), located ventral to the antennal lobe; (e) IR8a (+) neurons from the antenna innervate a group of glomeruli in the posterolateral and central region of the antennal lobe; while (f) Gr1 (+) neurons originating from the maxillary palps uniquely innervate a single large glomerulus, found in the posterior region of the antennal lobe. Maximum intensity projections ( $d-e$ ) and a single posterior z-slice (f) for the anterior view of adult female mosquito brains are shown at 10X magnification. The antennal lobes are encircled in white. Arrows indicate expression of the $3 x P 3-E C F P$ marker in the outer optic lobes. 
a<smiles>CC(C)(C)C(C)(C)C</smiles>

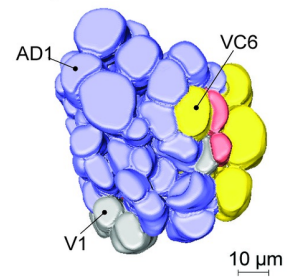

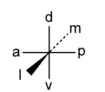

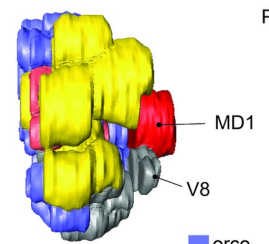

$\square$ orco$$
\text { b }{ }^{10000}
$$

Figure 2. $\mathrm{CO}_{2}$ receptor complex neurons innervate the largest glomerulus in the Aedes aegypti antennal lobe.
(a) 3D reconstructed model of the left antennal lobe of a female $A$. aegypti mosquito as seen from the anterior, lateral, posterior and medial perspectives. On this model, 60 glomeruli innervated by orco (+) neurons are shaded blue, 15 glomeruli receiving projections from IR8a $\left(^{+}\right)$neurons are shaded yellow, the MD1 glomerulus targeted by Gr1 (+) neurons is shaded red. A group of 8 ventral glomeruli not innervated by any of the three classes of chemosensory neurons are shaded grey. Glomeruli putatively co-innervated by orco (+) and IR8a (+) neurons are shaded orange. Eight landmark glomeruli for spatial registration are shown on the model. These include AD1 and VC6, seen on the anterior surface, and MD1, V1, V8, PL9, CD4 and D5 on the posterior surface. Scale bar for 3D model: $10 \mu \mathrm{m}$. Template genotype: orco ${ }^{Q F 2}>30 X Q U A S-m C D 8:: G F P$. (b) Average volumes for 75 glomeruli that were consistently identified in antennal lobe reconstructions. Three glomeruli (D6, VC11 and VC12) could not be consistently identified across all reconstructions and thus were excluded from this volumetric analysis. Based on observed levels of GFP fluorescence throughout individual glomeruli, we classified the innervation pattern as being 'heavy' or 'sparse' with the latter glomeruli labeled by asterisks. MD1 innervated by $\mathrm{Gr} 1(+)$ neurons is the largest antennal lobe glomerulus with a mean volume of approximately $6000 \mu \mathrm{m}^{3}$. Mean volumes $+/$ - s.e.m. are plotted, $n=5$ brains. 

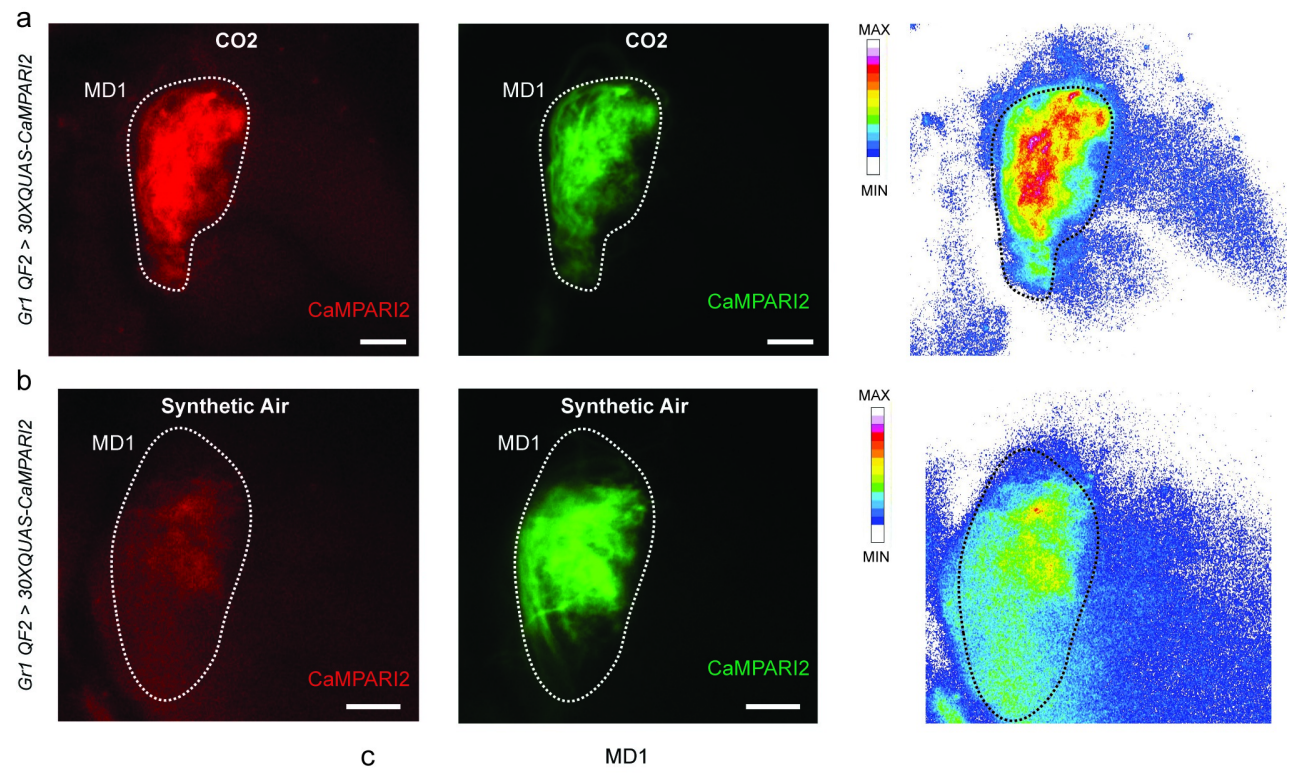

MD1

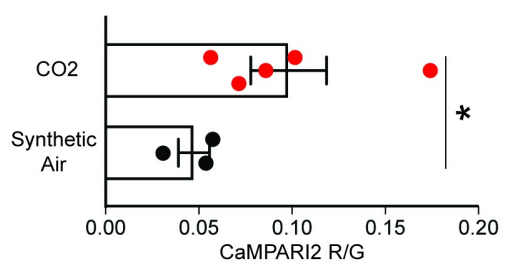

Figure 3: The Aedes aegypti MD1 glomerulus detects $\mathrm{CO}_{2}$. Female $\mathrm{Gr} 1^{\mathrm{QF} 2}>30 X C a M P A R / 2$ mosquitoes were exposed to simultaneous pulses of $1 \% \mathrm{CO} 2$ and $405 \mathrm{~nm}$ photoconversion light, and post-stimulation brains were dissected to analyze activity-dependent neural labeling visualized by the ratiometric intensity of red to green CaMPARI2 fluorescence. (a) The MD1 glomerulus, receiving innervation from Gr1 (+) maxillary palp neurons was strongly activated by $\mathrm{CO}_{2}$, in contrast to (b) weak activity labeling in response to stimulation with synthetic air. (c) Mean CaMPARI2 photoconversion values were significantly different between $\mathrm{CO}_{2}$ and control synthetic air conditions $\left(P=0.037^{*}\right.$, 453 Mann-Whitney test, $n=3-5$ brains per stimulus, mean R/G values $+/$ - s.e.m. plotted). Green and Red CaMPAR/2 signal in MD1 are shown after odor stimulation. Red CaMPARI2 in IR8a (+) glomeruli is visualized on the right panels as a heatmap of red fluorescence intensity. Dotted lines outline the boundary of the MD1 glomerulus. MD1 from the left antennal lobe was imaged at $63 \mathrm{X}$ magnification. Scale bars: $10 \mu \mathrm{m}$. 

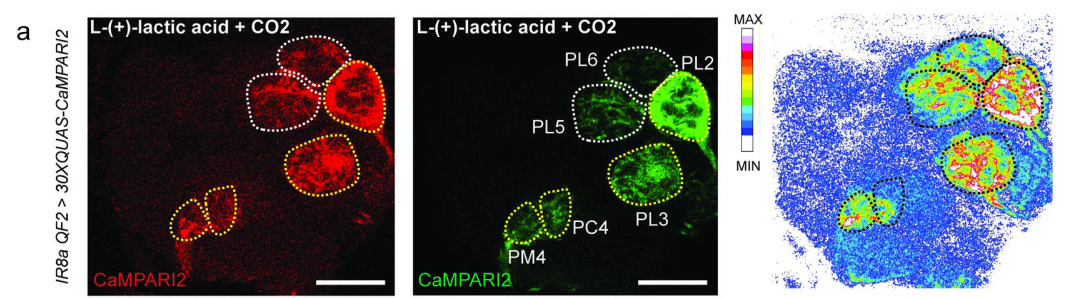

b
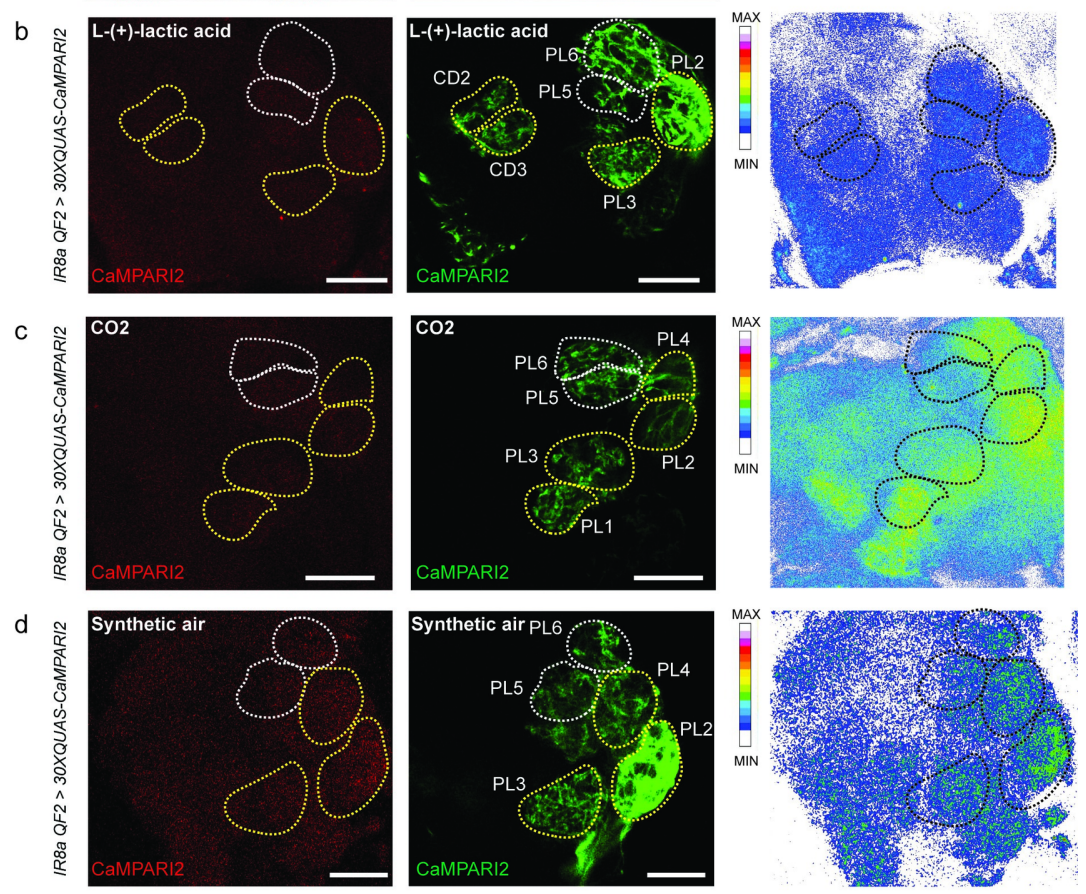

e
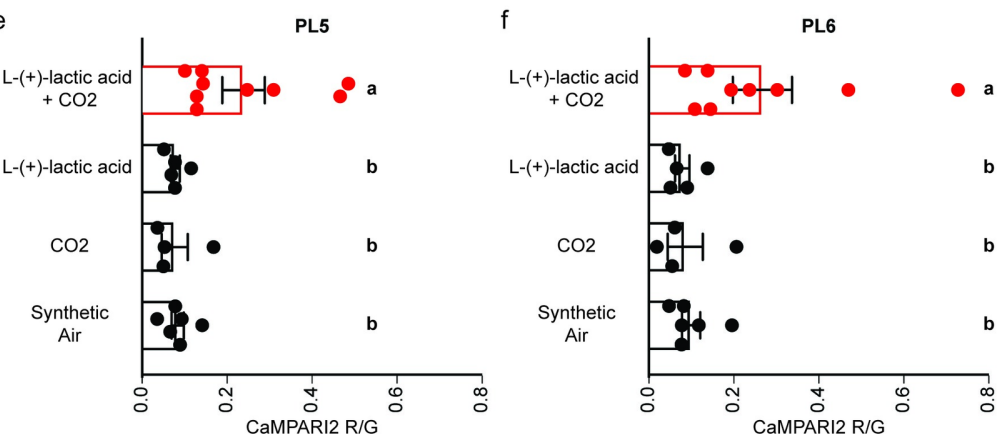

Figure 4: $\mathrm{CO}_{2}$ and L-(+)-lactic acid co-stimulation synergistically gates pre-synaptic calcium levels in acidsensing IR8a neurons. OSN axon terminals of IR8a (+) neurons innervating the antennal lobe exhibited strong CaMPARI2 photoconversion upon co-stimulation of female IR8a ${ }^{Q F 2}>30 X C a M P A R / 2$ mosquitoes with simultaneous pulses of $1 \% \mathrm{CO}_{2}$ with L-(+)-lactic acid (a). In contrast mosquitoes stimulated with either odorant alone (b - c), showed minimal photoconversion above background levels (d). Glomeruli PL5 and PL6 were more significantly photoconverted in the presence of $\mathrm{L}-(+)$-lactic acid and $\mathrm{CO}_{2}$ relative to all other odor treatments $(\mathbf{e}-\mathbf{f})(P<0.05$ for all comparisons to $\mathrm{L}-(+)$-lactic acid $+\mathrm{CO}_{2}$, Tukey's Multiple Comparison Test, $n=4-9$ brains per stimulus, mean R/G values + -- s.e.m. plotted). Additional glomeruli in the same z-plane were also noticeably activated in some replicates (see Fig. S9). Red CaMPARI2 in IR8a (+) glomeruli is visualized on the right panels as a heatmap of red fluorescence intensity. Dotted outlines represent the boundaries of the IR8a (+) glomeruli in these representative z-slices. The IR8a (+) glomeruli from the left antennal lobe were imaged at $63 \mathrm{X}$ magnification. Scale bars: $10 \mu \mathrm{m}$. 


\section{SUPPLEMENTARY MATERIALS}

472

\section{Materials and Methods:}

474

\section{Mosquito Stock Maintenance}

476 The Aedes aegypti LVPib12 strain (55) was used as the recipient genetic background for the

477 generation of all transgenic lines and subsequent assays. Mosquitoes were maintained with a 12

$478 \mathrm{hr}$ light:dark photoperiod at $27^{\circ} \mathrm{C}$ and $80 \%$ relative humidity using a standardized rearing protocol

479 (17). All experiments were conducted with non-blood fed and mated $A$. aegypti females that were

$480 \quad 5-10$ day old. Adult mosquitoes were provided constant access to a $10 \% \mathrm{w} / \mathrm{v}$ sucrose solution.

\section{Selection and in vitro transcription of sgRNAs}

Single guide RNA (sgRNA) target sites in the coding sequences of orco (AAEL005776), IR8a (AAEL002922) and Gr1 (AAEL002380) were identified using online design pipelines at http://zifit.partners.org/ZiFiT/ and http://crispr.mit.edu/. Candidate sgRNAs at each locus were prioritized for downstream use based on their putative lack of off-target activity in the $A$. aegypti genome. sgRNAs were transcribed and purified according to the method of Kistler et al. (2016) (32). Briefly, DNA templates for sgRNA synthesis were generated by PCR with two partially overlapping PAGE-purified oligos (IDT) for each target. sgRNA was subsequently produced using the MegaScript T7 in vitro transcription kit (Ambion) and purified using the MEGAclear transcription clean-up kit (Invitrogen). Prior to microinjection, sgRNA activity was confirmed by in vitro cleavage assays with purified recombinant Cas9 protein (PNA Bio, Inc., CP01-200) following the manufacturer's instructions. See Table S1 for final sgRNA sequences.

\section{T2A-QF2 Donor Constructs}

496 A base T2A-QF2 donor construct (pBlackbird) for CRISPR-Cas9 mediated homologous 497 recombination into target chemoreceptor loci in $A$. aegypti was generated by sequential rounds 498 of In-Fusion cloning (TakaraBio). This construct was generated with a Swal site for in-frame 499 insertion of the $5^{\prime}$ homology region from a of a gene directly with the T2A-QF2 coding sequence.

500 To survey for homology arms, genomic DNA regions spanning each target site were first PCR 501 amplified with CloneAmp (TakaraBio) using the following primers for orco (5'502 TGCAAGTGGATCATTTGTCG-3' and 5'-GTGCAATTGTGCCATTTTGA-3'), IR8a (5'503 CAAAGTATAATTTCGCCCCCTCC-3' and 5'-CTCTATGGCAGCCAAGATATTGG-3') and Gr1 504 (5'-AAGCCAGCTGGAAGGACATA-3' and 5'-ACCGTTTGGAGGTTGAATTG-3'). PCR products 
were cloned into pCR2.1-TOPO (Invitrogen) for subsequent sequence verification. After determining the most common sequence clone for each region, homology arms flanking the

507 CRISPR-Cas9 cut site were then PCR-amplified and inserted into the pBlackbird donor at the 508 Swal site $\left(5^{\prime} \mathrm{arm}\right)$ and BssHII site ( $\left.3^{\prime} \mathrm{arm}\right)$ using the In-Fusion primers listed in Table S2, to 509 generate a T2A-in frame fusion into the coding exon of interest. Three donor constructs that 510 yielded successful integrations at these target loci included pBlackbird-AaOrco-sg2, pBlackbird-

511 AalR8a-sg2 and pBlackbird-AaGr1-sg2. Each T2A-QF2 donor construct included a floxed 3xP3-

512 DsRed2 cassette as transformation marker, as well as a 3xP3-ECFP cassette in the vector 513 backbone outside the transposition cassette as a marker to assess putative ends-in recombination events at the target locus or alternate off-target integrations elsewhere in the genome.

\section{Mos1 mariner QUAS Reporter and Germline Cre Constructs}

QUAS reporter and germline Cre cassettes were generated by sequential rounds of In-Fusion cloning (TakaraBio) into template plasmid backbones for Mos1 mariner transposition (pMOS3xP3-ECFP and pMOS-3xP3-dsRed) (33) as outlined in Table S3. All QUAS reporter constructs included a 3XP3-ECFP cassette to mark transformants, while the pMOS backbone for QUASCaMPAR/2 was modified to remove the existing 3xP3-DsRed2 cassette from that vector and replace it with floxed $3 x P 3-E C F P$ cassette. The pMOS backbone for generating exu-Cre was modified to have a Polyubiquitin-EYFP marker using standard cloning methods. Final plasmids

525 that yielded transformants included: pMosECFP-QUAS-mCD8::GFP-p10, pMosIECFP-QUASCaMPARI2-p10 and pMosEYFP-Exu-Cre-p10.

The complete nucleotide sequences for all donor plasmids, pMOS vector backbones and Mos1 helper (33) plasmids used in this study will be deposited to Addgene, and template materials are listed in Table S3.

\section{Generation of Transgenic Lines}

533 T2A-QF2 knock-in lines into orco, Ir8a and Gr1 were generated via CRISPR-Cas9 mediated 534 homologous recombination (32) using embryonic microinjection.

536 To generate the $G r 1^{Q F 2 R e d}$ insertion, an injection mixture consisting of sgRNA (40ng/ul), purified 537 recombinant Cas9 protein (PNA Bio, 300ng/ul) and donor plasmid (500ng/ul) was prepared in microinjection buffer $(5 \mathrm{mM} \mathrm{KCl}$ and $0.1 \mathrm{mM} \mathrm{NaH} 2 \mathrm{PO} 4, \mathrm{pH} 7.2)$; and microinjected into the 
posterior pole of pre-blastoderm stage LVPib12 embryos of at the Insect Transformation Facility at University of Maryland (UM-ITF) using standard methods.

542 To generate the orco ${ }^{Q F 2 R e d}$ and IR8a $a^{Q F 2 R e d}$ insertions, for each target in vitro transcribed sgRNA 543 (100ng/ul) was mixed T2A-QF2 donor construct (100 ng/ul) and microinjected in the McMeniman 544 laboratory into the posterior pole of transgenic $A$. aegypti pre-blastoderm stage embryos 545 expressing Cas 9 under the maternal germline promoter exuperantia (56). Transformed $\mathrm{G}_{1}$ larvae

546 from all knock-in lines were isolated via the visible expression of 3xP3-DsRed2 fluorescent marker 547 in eye tissue and were outcrossed to the LVPib12 wild-type line for at least five generations prior 548 to attempting to generate homozygous strains. Precise insertion of each donor construct was confirmed by PCR amplification and subsequent Sanger sequencing of regions covering the homology arms and flanking sequences on either side of the insertion.

QUAS reporter and exu-Cre strains were generated by co-injecting each pMOS donor construct (500 ng/ul) with a pKhsp82 helper plasmid (300 ng/ul) expressing the Mos1 transposase (33) to foster quasi-random integration into the genome. Embryo microinjections were carried out by UMITF using standard techniques. For QUAS reporters, $\mathrm{G}_{1}$ lines were selected for stock establishment that had the strongest $3 x P 3-E C F P$ expression levels in the eyes and ventral nerve cord, indicative of responder loci accessible for neuronal expression.

558

\section{Cre-LoxP Mediated Excision of 3xP3 Fluorescent Markers}

560 To remove the floxed $3 x P 3-D s R e d 2$ cassette from each driver line (IR8a ${ }^{Q F 2 R e d}$, orco ${ }^{Q F 2 R e d}$, $561 G r 1^{Q F 2 R e d}$ ), we crossed males of each 3xP3-DsRed2 marked QF2 driver line to females of the 562 exu-Cre line we generated. We then screened $F_{1}$ progeny for loss of the DsRed2 marker. In the

563 case of the $G r 1^{\text {QF2Red }}$ line, the reduplicated marker due to the ends-in insertion was incompletely 564 removed in $\mathrm{F}_{1}$ progeny, so progeny lacking visible DsRed2 or ECFP markers were mated to their 565 exu-Cre (+) siblings to ensure complete excision of all markers. Precise excision was confirmed 566 for all three driver lines by PCR and Sanger sequencing using this strategy. Marker-free QF2 567 driver lines are denoted as $I R 8 a^{Q F 2}$, orco ${ }^{Q F 2}$ and $G r 1^{Q F 2}$.

568

\section{Mos1 mariner Splinkerette PCR}

570 QUAS and Exu-Cre transgenes inserted via Mos1 mariner transposition were mapped to 571 chromosomal locations (AaegL5.0 genome assembly) using a modified Splinkerette PCR, based 572 on the protocol described in Potter and Luo (2010) (57). Genomic DNA from single transgenic 
573 individuals was digested using the restriction enzymes BamHI-HF, Bglll, and BstYI (New England 574 BioLabs) in separate reactions; digests were left overnight ( 16 hrs). BstYl reactions were 575 subsequently heat-inactivated at $80^{\circ}$ for 20 minutes according to the recommended protocol. 576 BamHI reactions were purified using the QIAquick PCR Purification Kit (QIAgen) according to 577 manufacturer instructions and eluted in $50 \mu \mathrm{H}_{2} \mathrm{O}$ after 4 minutes of incubation at $50^{\circ} \mathrm{C}$.

Digests of genomic DNA were ligated to annealed SPLNK oligos as described (57). Splinkerette oligonucleotides 5'-GATCCCACTAGTGTCGACACCAGTCTCTAA-TTTTTTTTTTCAAAAAAA-3'

AATGAGACTGGTGTCGACACTAGTGG-3' were first annealed and ligated to digested genomic DNA. The first- and second-round PCR amplification steps were modified, using the standard SPNLK primers and new primers designed to the inverted repeat regions of the Mos1 mariner transposon. PCR products were amplified using Phusion High-Fidelity DNA Polymerase (NEB).

First round Splinkerette PCR was carried out using the primers 5'CGAAGAGTAACCGTTGCTAGGAGAGACC-3' and 5'-TCAGAGAAAACGACCGGAAT-3' for the right inverted repeat, and 5'-CGAAGAGTAACCGTTGCTAGGAGAGACC-3' and 5'CACCACTTTTGAAGCGTTGA-3' for the left inverted repeat. The second round of Splinkerette PCR was carried out using the primers 5'-GTGGCTGAATGAGACTGGTGTCGAC-3' and 5'TCCGATTACCACCTATTCGC-3' for the right inverted repeat, and 5'GTGGCTGAATGAGACTGGTGTCGAC-3' and 5'-ATACTGTCCGCGTTTGCTCT-3' for the left inverted repeat. In the case of QUAS-CaMPAR/2, the extension time of the second-round PCR was lengthened to 4 minutes to amplify longer segments of flanking DNA. PCR products were gel purified and Sanger sequenced with additional sequencing primers for the right (5'AAAAATGGCTCGATGAATGG-3') and left (5'-GGTGGTTCGACAGTCAAGGT-3') inverted repeats. BLAST searches were used to map Splinkerette fragments derived from each Mos1 mariner cassette to coordinate locations in the genome at canonical TA dinucleotides (58) and insertion sites (Table S5) were subsequently confirmed by PCR.

601

\section{Genotyping Gr1 ${ }^{\text {QF2Red }}$ and Gr1 ${ }^{\text {QF2 }}$}

$603 \mathrm{Gr} 1^{\text {QF2Red }}$ and $\mathrm{Gr} 1^{\text {QF2 }}$ knock-ins were genotyped using a multi-primer PCR assay with the forward 604 primer: 5'-CATGTACATCCGCAAGTTGG-3'; and two standard reverse primers: 5'605 TGTTAGTGAGATCAGCGAACCT-3' and 5'-GATCAACCCACAGATGACGA-3'. Fragments for 606 size-based genotyping were amplified via DreamTaq (Thermo Scientific) and analyzed by 
607

608

609

610

611

612

613

614

615

616

617

618

619

620

621

622

623

624

625

626

627

628

629

630

631

632

633

634

635

636

637

638

639

640

conventional agarose gel electrophoresis. Each of the reverse primers were used at half the normal concentration. This resulted in a single 689 bp amplicon in homozygous mosquitoes; a single 884 bp amplicon in wild-type mosquitoes; and two amplicons, one at 689 bp and one at $884 \mathrm{bp}$, in heterozygous mosquitoes.

\section{Genotyping IR8a $a^{Q F 2 R e d}$ and IR8a $a^{Q F 2}$}

$I R 8 a^{Q F 2 R e d}$ and $I R 8 a^{Q F 2}$ knock-ins were genotyped using a multi-primer PCR assay with the forward primer: 5'-AGGAGATTGCGCTTGTCCTA-3'; and two standard reverse primers: 5'CCCCGACATAGTTGAGCATT-3' and 5'-TGTTAGTGAGATCAGCGAACCT-3'. Each of the reverse primers were used at half the normal concentration. This resulted in a single $560 \mathrm{bp}$ amplicon in homozygous mosquitoes, a single $501 \mathrm{bp}$ amplicon in wild-type mosquitoes, and two amplicons, one at $560 \mathrm{bp}$ and one at $501 \mathrm{bp}$, in heterozygous mosquitoes.

\section{Genotyping orco ${ }^{Q F 2 R e d}$ and orco $^{Q F 2}$}

orco $^{\text {QF2Red }}$ and orco ${ }^{Q F 2}$ knock-ins were genotyped using conventional PCR. The PCR reaction used the forward primer: 5'-GCGATAGCGTCAAAAACGTA-3' and reverse primer: 5'ATTCCTTGAAGGTCCATTGCAG-3'. This resulted in an 1842 bp amplicon corresponding to the orco $^{\text {QF2 }}$ allele, a $3129 \mathrm{bp}$ amplicon corresponding to the orco ${ }^{\text {QF2Red }}$ allele, and/or a $367 \mathrm{bp}$ amplicon corresponding to the wild-type allele. Heterozygotes had both wild-type and transgenic PCR bands.

\section{Genotyping QUAS-mCD8:GFP}

QUAS-mCD8:GFP-11F4 was genotyped using conventional PCR. The PCR reaction used the forward primer: 5'-TCCAGCCGATAGGAACAATC-3' and reverse primer: 5'CAAATCCGAATTTCCCGTAA-3'. This resulted in a single 5797 bp amplicon for homozygotes and a 444 bp for the wild-type allele. Heterozygotes typically only had the wild-type PCR band.

\section{Genotyping QUAS-CaMPARI2-F2}

QUAS-CaMPAR/2-F2 was genotyped using a multi-primer PCR assay with the forward primer: 5'-GTTTGACCAAATGCCGTTTC-3'; and two standard reverse primers: 5'GTCGATAGGCGCGTAGTGTA-3' and 5'-CACCACTTTTGAAGCGTTGA-3'. Each of the reverse primers is used at half the normal concentration. This results in a single $645 \mathrm{bp}$ amplicon in homozygous mosquitoes, a single 874 bp amplicon in wild-type mosquitoes; and two amplicons, one at $645 \mathrm{bp}$ and one at $874 \mathrm{bp}$ in heterozygous mosquitoes. 
641 Transgenic Stock Maintenance and Composite Genotypes

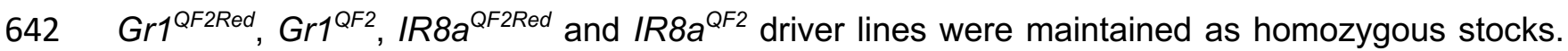

$643 \operatorname{orcoQ}{ }^{F 2 R e d}$ was maintained as a heterozygous stock by outcrossing to LVPib12 each generation.

644 orco $^{Q F 2}$ was maintained as a heterozygous stock by outcrossing to either LVPib12 or QUAS-

$645 m C D 8:: G F P$ each generation and screening for GFP fluorescence in the olfactory tissues of the

646 progeny. 15xQUAS-mCD8::GFP and 15xQUAS-CaMPAR/2 responder lines were maintained as

647 homozygous stocks. The exu-Cre line was maintained as a heterozygous stock by outcrossing to

648 LVPib12 each generation. Stock and composite genotypes used in each figure panel are detailed

649 in Table S4. Cytogenetic locations of all transgenes generated in this study are detailed in Figure

650 S10. Cytogenetic locations of all transgenes generated in this study are detailed in Figure S11.

651

652

Immunohistochemistry

653 Immunostaining of female $A$. aegypti brains was performed as previously described (17), with

654 minor modifications. Briefly, severed mosquito heads were fixed in $4 \%$ paraformaldehyde

655 (Milonig's buffer, pH 7.2) for three hours and brains were carefully dissociated from the head 656 capsule, pigmented ommatidia and air sacks. Dissected brains were then subjected to three 20 657 min washes at room temperature in PBST (0.1M PBS with 0.25\% Triton-X 100), and allowed to 658 incubate overnight in a blocking solution consisting of $2 \%$ normal goat serum (NGS) and $4 \%$ 659 Triton-X 100 in $0.1 \mathrm{M} \mathrm{PBS}$ at $4^{\circ} \mathrm{C}$. Brains were then washed three times for 20 min each in PBST 660 and incubated for three days at $4^{\circ} \mathrm{C}$ in a primary antibody solution containing mouse anti-BRP 661 (DSHB, nc82-s, AB_2314866, 1:50 v/v) targeting the pre-synaptic active zone protein Bruchpilot 662 (41) and rabbit anti-GFP (Invitrogen, A-6455, 1:100 v/v) targeting $m C D 8:: G F P$. Brains were then 663 washed three times for $20 \mathrm{~min}$ each in PBST and incubated for 3 days at $4^{\circ} \mathrm{C}$ in a secondary 664 antibody solution consisting of goat anti-mouse Cy3 (Jackson ImmunoResearch, AB_2338680,

$6651: 200 \mathrm{v} / \mathrm{v}$ ) and goat anti-rabbit Alexa Fluor 488 (Invitrogen, A-11008, 1: $200 \mathrm{v} / \mathrm{v}$ ). All primary and 666 secondary antibody dilutions were prepared in PBST with $2 \% \mathrm{v} / \mathrm{v}$ NGS. Brains were finally washed 667 three times for 20 min each in PBST at room temperature and mounted in 20 ul of Slow-Fade 668 Gold Antifade Mountant (Invitrogen, S36936) on glass slides with coverslip bridges (Number 2$669170 \mu \mathrm{m})$.

670

671 Immunohistochemistry Image Acquisition Settings

672 Brain immunostaining images were acquired on a single-point laser scanning Carl-Zeiss LSM 780 673 confocal microscope. To capture images of the entire adult brain, a 10X objective lens (0.3 NA, 674 Plan-Apochromat) was used. Excitation of Cy3 signal was achieved with a $561 \mathrm{~nm}$ solid-state 
675 laser line at $0.05 \%$ laser power, and GaAsP detector gain set to 825; while a $488 \mathrm{~nm}$ laser line 676 was used to excite Alexa Fluor 488 (20\% laser power, detector gain at 825$)$. We additionally 677 acquired images with a 20X objective lens (0.8 NA, Plan-Apochromat) to perform 3D 678 reconstructions of the antennal lobes. For these the power of the $488 \mathrm{~nm}$ laser line was adjusted 679 to $5 \%$. For each antennal lobe, 60 z-slices with a z-step size of $1 \mu \mathrm{m}$ and a $1024 \times 1024-$ pixel 680 resolution were acquired.

681

\section{Antennal lobe reconstructions}

683 3D morphological reconstructions of left antennal lobes were performed as previously described 684 (17). Briefly, confocal images were imported in *.Ism format into Amira (FEI Houston Inc) and then segmented by highlighting all pixels across a z-stack occupied by individual glomeruli. The nc82 channel was used for manual segmentation of individual glomeruli. The GFP channel was then used to identify orco, IR8a and Gr1-positive glomeruli. To name glomeruli we identified landmark glomeruli in each antennal lobe sample and using a systematic antennal lobe reference key (17) we then designated names to all GFP labeled glomeruli based on their spatial positions relative to the landmarks. 3D and 2D antennal lobe models were generated by surface rendering.

691 Glomerular volumes were obtained $\left(\mu \mathrm{m}^{3}\right)$ from the left antennal lobe of five replicate brains using 692 the nc82 channel.

693

\section{Imaging of Peripheral Olfactory Appendages}

695 Live antenna, palp and proboscis tissue were dissected in 0.1M PBS and immediately mounted 696 in Slow-Fade Gold Antifade Mountant (Invitrogen, S36936). Images were acquired on a Carl697 Zeiss LSM 780 confocal microscope within 1 hour of dissection. To excite the GFP signal, the $698488 \mathrm{~nm}$ laser line was used at 5\% laser power. An additional DIC channel was used to visualize 699 gross morphology of the peripheral tissue. Images of the antennae were acquired with a 20X objective lens (0.8 NA, Plan-Apochromat), while images of the palp and labella of the proboscis were acquired with a 40X (1.3 NA, Plan-Apochromat) oil immersion objective.

\section{Live Mosquito Preparation for CaMPAR/2 Photoconversion}

704 To prepare mosquitoes for CaMPARI2 photoconversion (47), mosquitoes were cold anesthetized 705 and tethered to an imaging chamber. To do this, the thorax of a female mosquito was first affixed 706 to the ventral surface of a 35mm petri dish lid (Eppendorf, 0030700112) using UV-curing adhesive 707 (Bondic) immediately proximal to a $15 \mathrm{~mm}$ diameter circular hole made in the lid center. Two 708 additional drops of adhesive were applied to the ommatidia on extremities of the mosquito head, 
to prevent head movement. A small piece of clear tape (Duck EZ Start, Heavy Duty Packaging Tape) was then affixed over the center hole such that the dorsal surface of the mosquito head could be gently affixed to the ventral adhesive tape surface. An excised section of plastic coverslip $(5 \mathrm{~mm} \times 3 \mathrm{~mm})$ was then affixed to the tape and used to shield the antennae from the adhesive tape surface and suspend these sensory appendages in the air.

The imaging chamber with head fixed mosquito was then inverted and a rectangular incision window approximately $400 \mu \mathrm{m} \times 200 \mu \mathrm{m}$ was cut through the tape window where the dorsal head cuticle and ommatidia were affixed. The wide boundary of the incision was typically made immediately adjacent to the first antennal subsegment along the lateral-medial brain axis, while the short boundary of the incision extended along the dorsal-ventral brain axis. To create this window, segments of ommatidia and bridge cuticle between the left and right eyes were gently incised and removed using a surgical stab knife (Surgical Specialties Corporation, Sharpoint, Part \# 1038016) to reveal the underlying antennal lobes. The exposed antennal lobes were then immediately immersed in an A. aegypti Ringer's solution (59) composed of $150 \mathrm{mM} \mathrm{NaCl}, 3.4 \mathrm{mM}$ $\mathrm{KCl}, 5 \mathrm{mM}$ glucose, $1.8 \mathrm{mM} \mathrm{NaHCO}_{3}, 1 \mathrm{mM} \mathrm{MgCl}$, 25mM HEPES and $1.7 \mathrm{mM} \mathrm{CaCl} 2$; $\mathrm{pH} 7.1$. Mosquitoes were allowed to recover for a period of $15 \mathrm{~min}$ from cold anesthesia and surgery in a humidified chamber at room temperature prior to imaging.

\section{CaMPARI2 Photoconversion}

For CaMPARI2 photoconversion, the tethered preparation was then placed under a 20X water dipping objective (Olympus XLUMPLFLN20XW, 1.0 NA) ensuring that the antennal lobes expressing basal green CaMPAR/2 signal were in focus. Each preparation was then exposed to a combined photoconversion-odor stimulation regime consisting of repetitive duty cycles of four $500 \mathrm{~ms}$ pulses of 405nm light from an LED driver (Thorlabs, DC4104, 1000mA current setting) synchronized with a 1 s odorant pulse as outlined in Fosque et al. 2015 (48), for 75 cycles with a total protocol duration of approximately 41 minutes.

\section{Odorant Delivery}

Pulses of odorants were delivered using a custom olfactometer device (Lundström et al 2010) with solenoid valves regulating delivery of odor stimuli from chambers equipped with pressuresensitive check valves (Smart Products USA, Inc.). $3 \mathrm{~mL}$ of control (dH2O) or treatment (L-(+)lactic acid solution, Sigma Aldrich, 27714) odors were placed into dedicated and sealed odor delivery vials. During 'odor onset', synthetic air (Airgas, AI UZ300) at a flow rate of $1 \mathrm{ml} / \mathrm{s}$ was 
passed through these holding chambers to carry headspace odors via Teflon tubing into a carrier airstream of humified synthetic air that was directed at the olfactory appendages of the mosquito using a plastic pipette. During CaMPARI2 photoconversion assays, the tethered mosquito preparation always received a constant amount of airflow $(5 \mathrm{ml} / \mathrm{s})$ during odor onset/offset from the stimulus pipette via solenoid valves simultaneously switching or combining humidified synthetic air, 5\% $\mathrm{CO}_{2}$ (Airgas, CD USP50) and L-(+)-lactic acid headspace as required for different odor treatments. In trials involving $\mathrm{CO}_{2}$, a $1 \mathrm{ml} / \mathrm{s}$ stream of $5 \% \mathrm{CO}_{2}$ was diluted $1: 5$ into the carrier airstream for a final concentration at the specimen of $1 \%$.

\section{CaMPARI2 Sample Processing}

Following photoconversion, the mosquito was gently untethered from the imaging chamber and the head severed and fixed in Milonig's buffer for 20 minutes. The brain was then dissected out in calcium-free Ringer's solution composed of $150 \mathrm{mM} \mathrm{NaCl}, 3.4 \mathrm{mM} \mathrm{KCl}, 5 \mathrm{mM}$ glucose, $1.8 \mathrm{mM}$ $\mathrm{NaHCO}_{3}, 1 \mathrm{mM} \mathrm{MgCl}$, $25 \mathrm{mM}$ HEPES and $10 \mathrm{mM}$ EGTA. To stain glomerular boundaries, we then incubated each brain in Alexa Fluor 647 Phalloidin (Invitrogen, A22287) prepared in calciumfree Ringer's solution (1:40 v/v dilution) for $30 \mathrm{~min}$. To prepare Alexa Fluor 647 phalloidin for use in imaging, first, a 400X DMSO stock solution was prepared according to the manufacturer's instructions by dissolving the fluorophore in 150 ul of DMSO. 1 ul of this DMSO stock was diluted in 399 ul calcium-free Ringer's solution to yield a 1X stock. This stock was then further diluted to a final concentration 1:40 in calcium-free Ringer's solution for staining. Brains were transferred directly from this solution into 20 ul of Slow-Fade Gold Antifade Mountant (Invitrogen, S36936) on glass slides with coverslip bridges (Number 2- $170 \mu \mathrm{m}$ ) for CaMPAR/2 and phalloidin imaging.

\section{CaMPARI2 Image Acquisition Settings}

Antennal lobes from CaMPARI2 photoconversion assays were imaged with a $63 \mathrm{X}(1.4 \mathrm{NA})$ oilimmersion objective on a Zeiss 880, Airyscan FAST super-resolution single point scanning microscope. Excitation of red CaMPARI2 signal was achieved with a $561 \mathrm{~nm}$ solid-state laser line at $14 \%$ laser power. Green CaMPARI2 was excited with a $488 \mathrm{~nm}$ argon laser line at $10 \%$ laser power. To visualize glomerular boundaries, a $633 \mathrm{~nm}$ diode laser was used to excite the Alexa647 phalloidin fluorophore at $40 \%$ laser power. Master detector gain was set to a value of 800 . We captured $0.987 \mu \mathrm{m}$ z-slices of 1572 X 1572-pixel resolution in the FAST mode. Raw images were further processed by applying the Airyscan method with 'auto' processing strength.

\section{CaMPARI2 Image Analysis}


777 Image analysis was carried out in Fiji (http://imagej.net/Fiji) and images were imported into the 778 program in the *.Ism format. We first applied a median filter (radius $=2$ pixels) to remove noise 779 and then a rolling ball subtraction (rolling ball radius $=80$ pixels), to correct for non-uniformity of 780 background intensities. We analyzed CaMPAR/2 photoconversion in the left antennal lobe of all 781 samples due the well-defined spatial arrangement and conspicuous boundaries of IR8a-positive 782 glomeruli in this lobe with phalloidin straining. ROls were defined by manually segmenting 783 glomeruli using the free hand selection tool. For IR8a glomeruli, we analyzed photoconversion 784 ratios for 12/15 IR8a-positive glomeruli that could be reliably identified across all AL samples. 785 These included: VC5, VC6, PL1-PL6, PM4, PC4, CD2 and CD3. The integrated density (mean 786 grey value $X$ area) for all $z$ - slices of the ROI, which included all representative slices of a target 787 glomerulus, was calculated in the green $(488 \mathrm{~nm})$ and red $(560 \mathrm{~nm})$ imaging channels. The final 788 measure of photoconversion, the red to green ratio $(R / G)$, was calculated as:

Glomeruli were named by co-localizing green and red CaMPARI2 signal to individual glomeruli evident in the Alex-Fluor 647 phalloidin channel and defining their spatial orientation relative to landmark and flanking glomeruli using our 2D CaMPAR/2-phalloidin antennal lobe reference map. 
a Orco QF2Red > 15XQUAS-mCD8::GFP

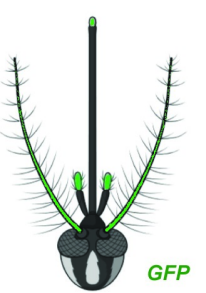

d

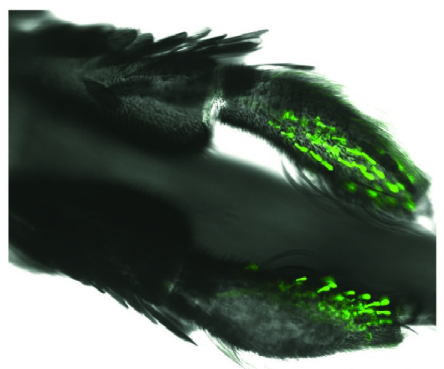

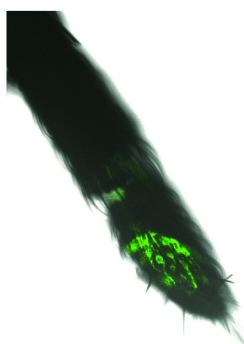

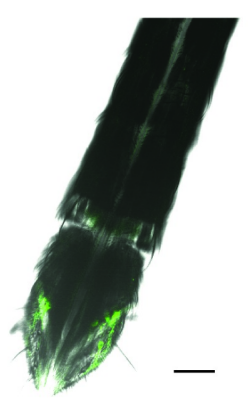

e

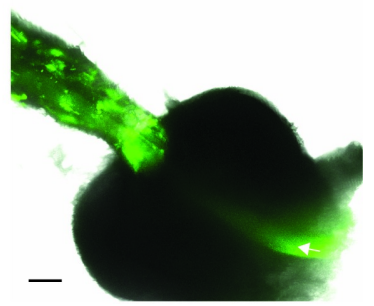

Figure S1. Additional peripheral innervation patterns of orco (+) olfactory sensory neurons on $A$. aegypti sensory appendages. (a) Schematic of the mosquito head showing the gross expression pattern from orCO $\mathrm{OF}^{\text {Red }}>$ 15XQUAS-mCD8::GFP females in peripheral olfactory tissue. (b-c) In addition to the third antennal segment shown in Figure 1, strong GFP expression from dorsal and ventral perspectives of the labella of the proboscis. Axonal projections of these neurons extend into the shaft of the proboscis. (d) orco expression was also strongly evident within capitate peg sensilla on the ventral surface of the maxillary palp. (e) Maximum intensity projection of a female pedicel at $40 \mathrm{X}$ magnification. No orco expression was noted in the scolopidia of the pedicel while the antennal nerve was observed to 


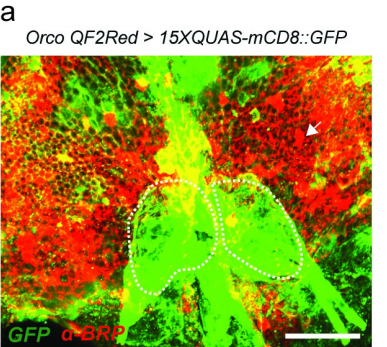

$\mathrm{d}$

Orco $Q F 2>30 X Q U A S-m C D 8:: G F P$

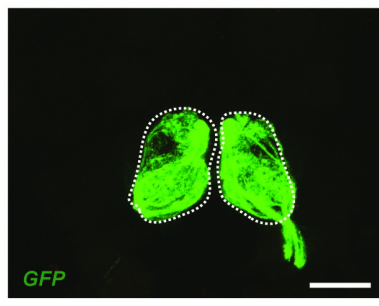

g Orco QF2 > 30XQUAS-mCD8::GFP

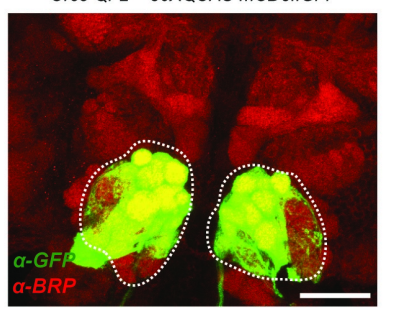

b

IR8a QF2Red > 15XQUAS-mCD8:.GFP

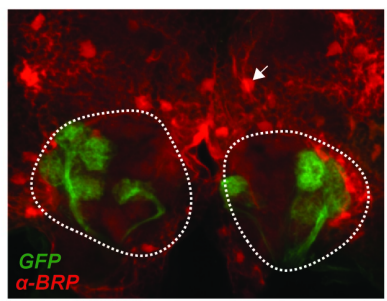

e

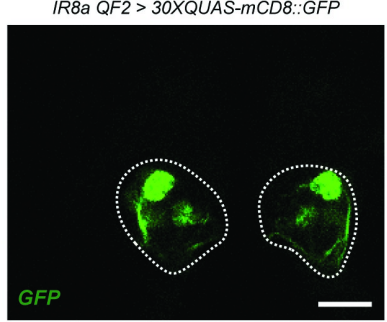

h IR8a QF2 > 30XQUAS-mCD8::GFP

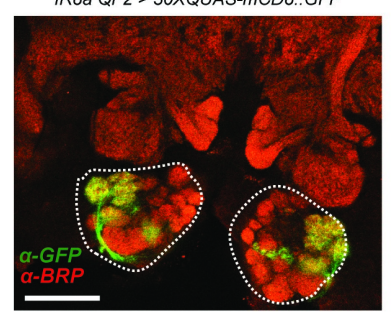

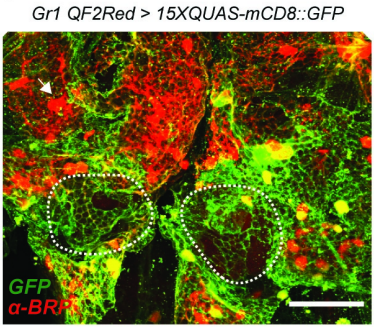

f

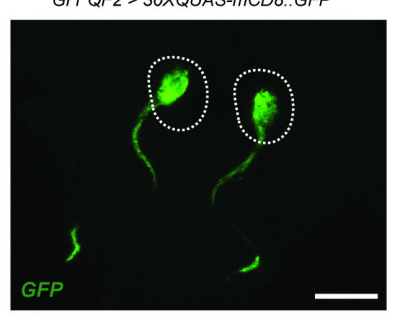

i $\quad$ Gr1 QF2 > 30XQUAS-mCD8::GFP

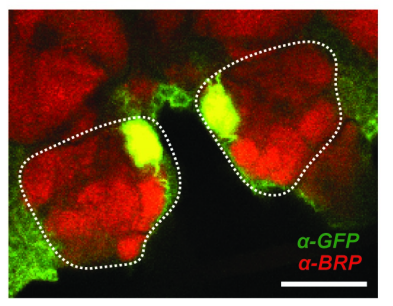

811 Figure S2. Promiscuous QF2/QUAS driven fluorescence in the $A$. aegypti brain and Cre-LoxP mediated

812 excision of $3 \times P 3$ marker cassettes. The transgenic $Q F 2^{R e d}$ driver lines described in this study carried a floxed $3 \times P 3-$

$813 D$ DRed2 cassette to mark successful transgenesis. However, upon close examination of the central brain we noted that

814 this marker cassette was not only expressed in the optic lobes, but was also strongly expressed in hexagonal cells

815 ensheathing the central brain which are putatively glia (arrows). In crosses involving the orco ${ }^{Q F 2 R e d}$ and Gr1 ${ }^{\text {QF2Red }}$ driver

816 lines, we also noted conspicuous green expression in these same locations suggesting the $3 \times P 3$ promoter was

817 influencing the expression pattern of the integrated T2A-QF2 transgene at these loci. (a-c) Anterior view of adult female

818 mosquito brains at 20X magnification. 3xP3 expression occludes the antennal lobes and other neuropil, in each of the

819 three driver lines, posing a significant challenge for confocal or two-photon imaging. Genetic excision of this marker

820 from the QF2 driver lines alone markedly improved our ability to clearly view the antennal lobes in both unstained (d-f)

821 and immunostained brain preparations (g-i). Scale bar: $50 \mu \mathrm{m}$. 


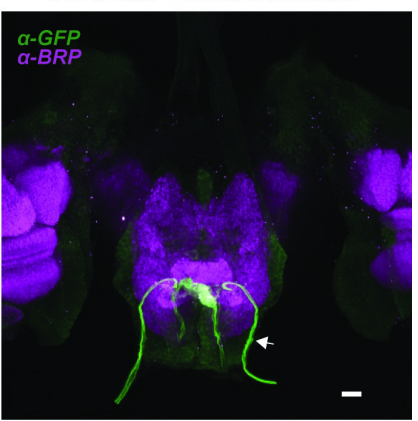

Figure S3. SEZ innervation of orco (+) neurons. Posterior view of the brain of an adult female orco ${ }^{Q F 2}>30 X Q U A S-$ $m C D 8:: G F P$ mosquito. orco (+) neurons from the labella project via the labial nerve (arrow) and terminate in the gustatory center of the mosquito brain, the suboesophageal zone (SEZ). In Anopheles gambiae, these terminal neuropil clusters were well separated and named SEZ glomeruli (Riabinina et al., 2016). In Aedes aegypti the boundaries of these clusters overlap and appear to be smaller in size. Scale bar: $20 \mu \mathrm{m}$. 
a

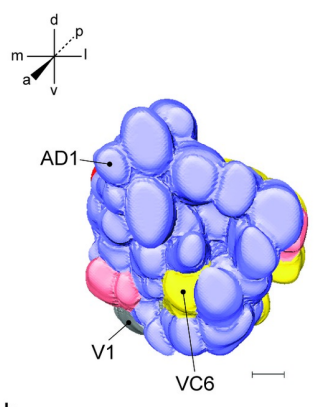

b

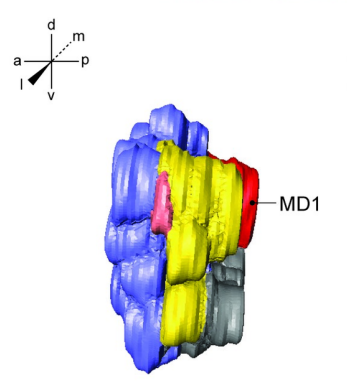<smiles>CC(C)(C)C(I)(I)I</smiles>
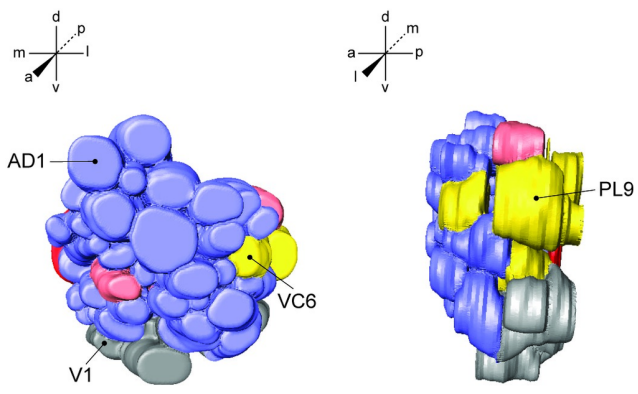

Gr1 QF2 > 30XQUAS-mCD8::GFP

IR8a QF2 > 30XQUAS-mCD8::GFP
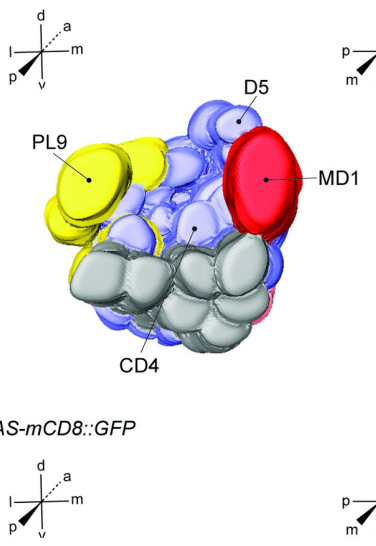

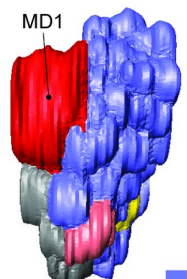

arco IR8a orco+IR8a $\square \mathrm{Gr} 1$

Figure S4. Three-dimensional models of the female antennal lobe from Ir8a and Gr1 driver lines. 3D
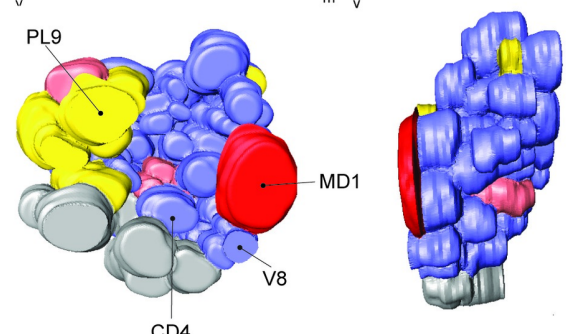
reconstructed models of the left antennal lobe from adult female Aedes aegypti from (a) IR8a ${ }^{\text {QF2 }}>30 X Q U A S$ $m C D 8:: G F P$ and (b) Gr1 ${ }^{\text {QF2 }}>$ 30XQUAS-mCD8::GFP genotypes are illustrated. 3D models are arranged (left to right) in the anterior, lateral, posterior and medial orientations. orco (+) glomeruli, IR8a (+) glomeruli, orco (+) / IR8a (+) glomeruli, and the Gr1 (+) (MD1) glomerulus are colored blue, yellow, orange and red respectively. Eight landmark glomeruli including AD1 and VC6 on the anterior surface, and MD1, V1, V8, PL9, CD4 and D5 on the posterior AL surface are labelled. Scale bar: $10 \mu \mathrm{m}$. These models are shown in 2D format in Figure S6 and S7. 

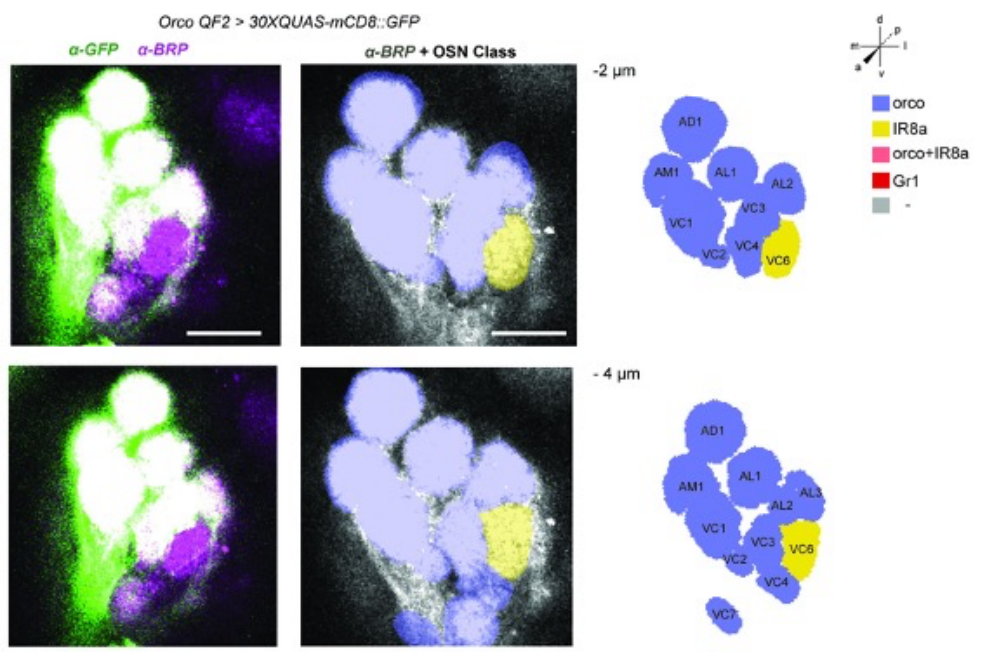

$.4 \mu \mathrm{m}$
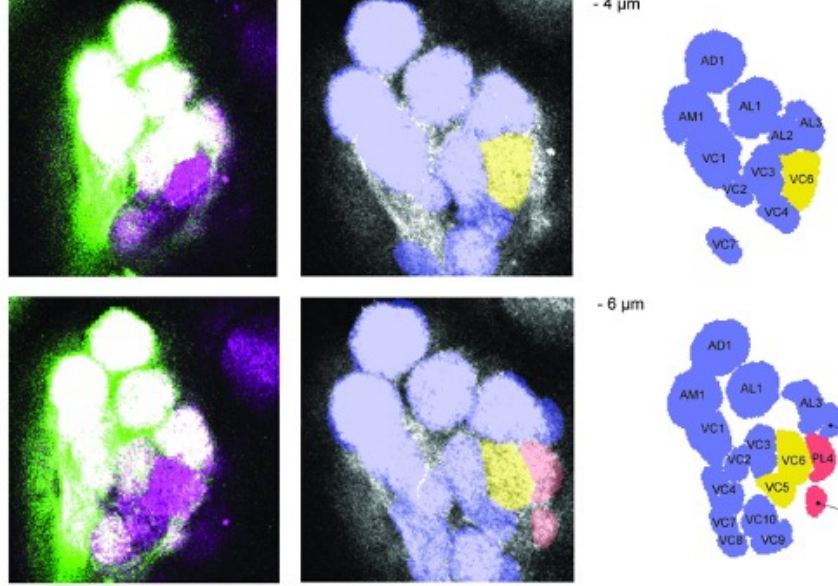

$.6 \mu \mathrm{m}$
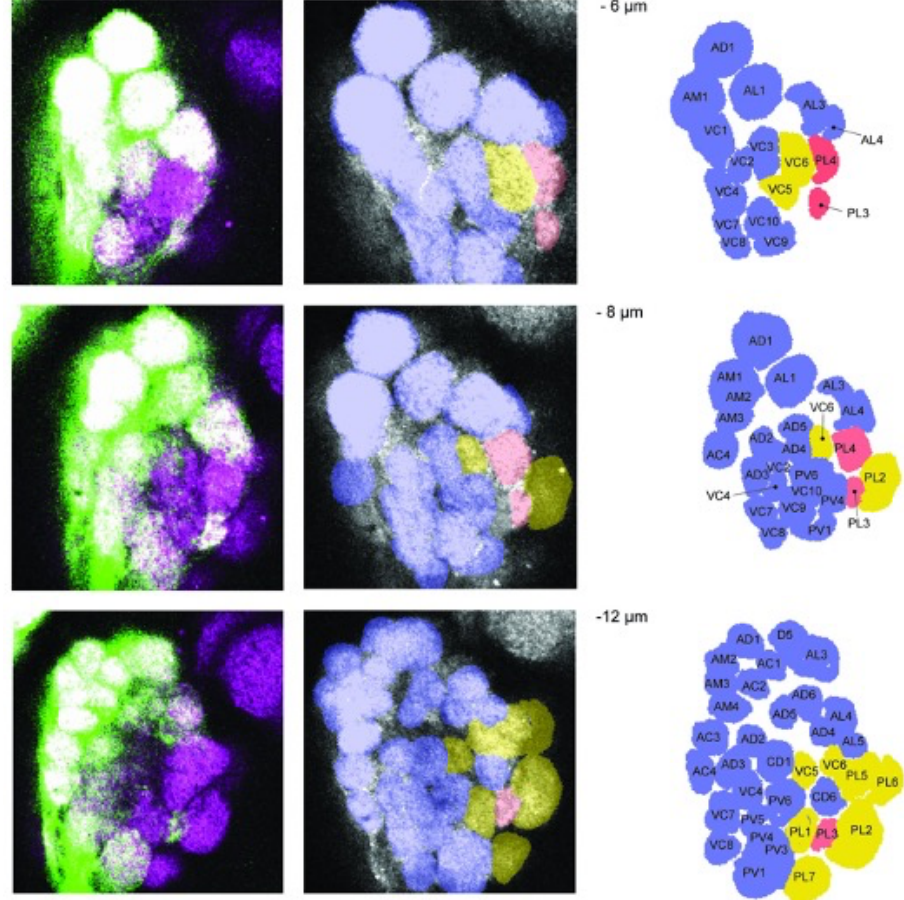

Figure S5. orco Receptor to Glomerulus 2D Map.

Representative confocal stack from the left antennal lobe of an adult female orco ${ }^{Q F 2}>30 X Q U A S-m C D 8:: G F P$ mosquito. The left panels show the antennal lobe at 20X magnification, with the GFP signal from orco neurons in green and the neuropil stained with BRP in magenta, to visualize glomerular boundaries. The middle panels show the BRP stained glomeruli overlayed with blue (orco+), yellow (Ir8a+), orange (putatively orco+ and Ir8a+), red (Gr1+), and grey colors (unknown chemosensory identity). The right panels show detailed spatial maps of all antennal glomeruli, glomeruli are color coded according to chemoreceptor class. 10 representative focal planes from a total of $60 \mathrm{z}$-slices are shown on this map. orco neurons innervate 60 glomeruli. These included all the anterior glomerular groups namely, the Antero-Dorsal (AD), Antero-Lateral (AL), Antero-Medial (AM), Antero-Central (AC) and the Ventro-Central (VC) groups. The posterior groups innervated by orco (+) neurons include the Postero-Medial (PM), Postero-Central (PC), Postero-Lateral (PL), Centro-Dorsal (CD), Postero-Ventral (PV), Dorsal (D) and Medio-Dorsal (MD) groups. A total of 49 spatially invariant glomeruli depicted in blue are shown in this 2D map. Scale bar: $10 \mu \mathrm{m}$. 
856
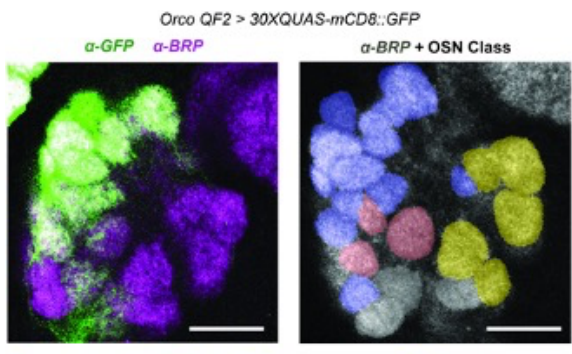

$-23 \mu \mathrm{m}$
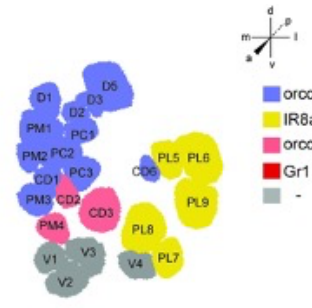

arco

IR8a

oroo+IR8a

a $\mathrm{Gr}$
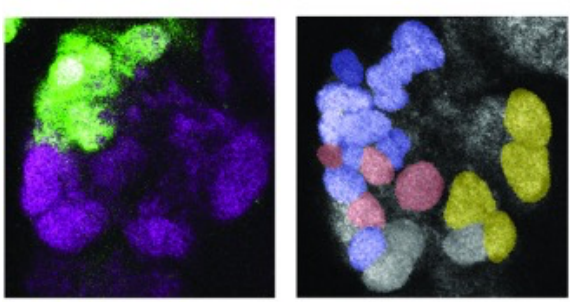

$-24 \mu \mathrm{m}$
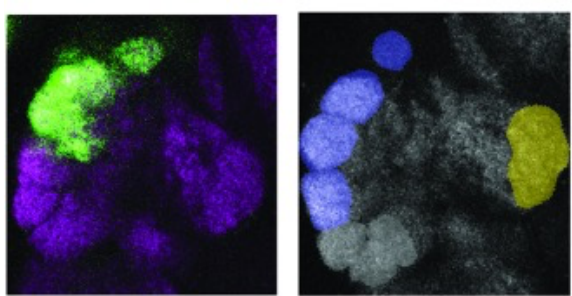

$.36 \mu \mathrm{m}$
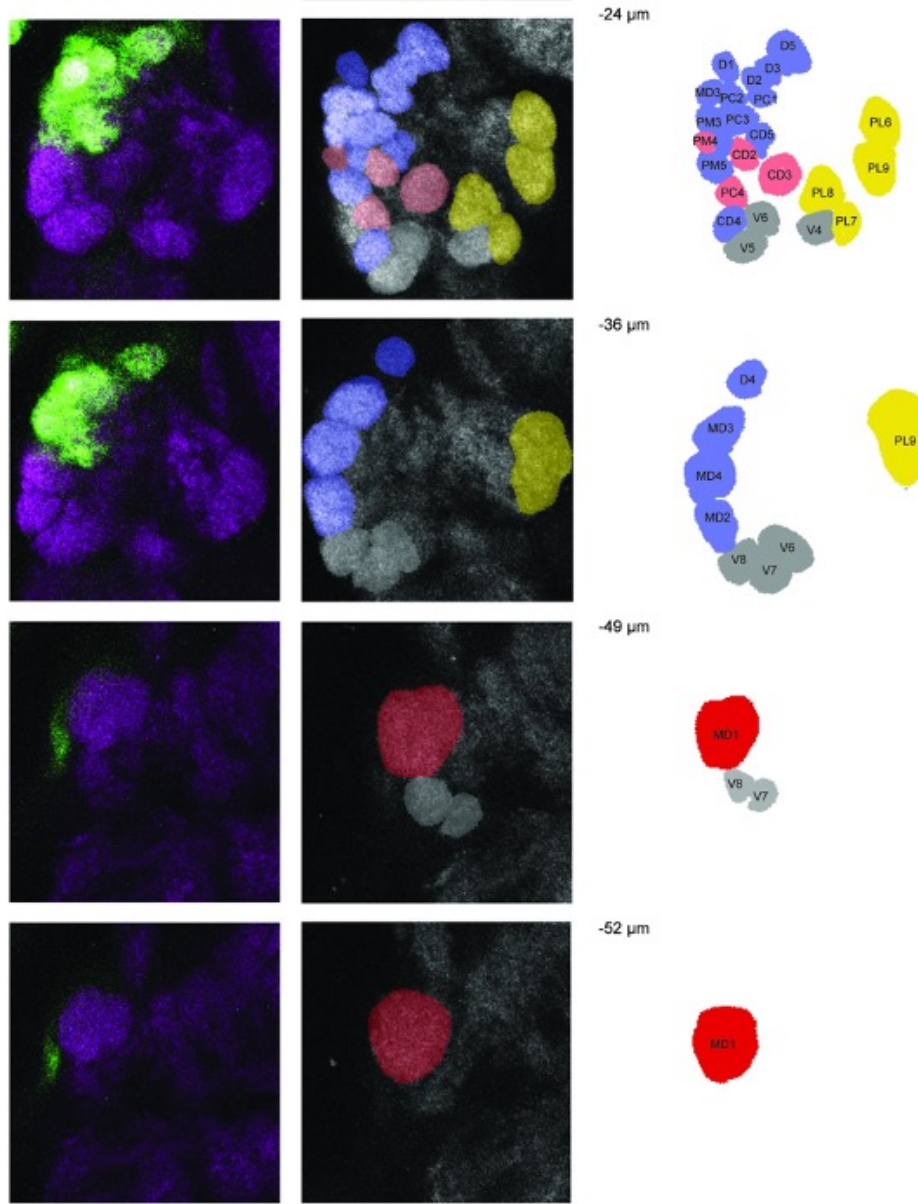

$-52 \mu \mathrm{m}$

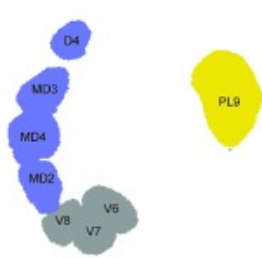

$-49 \mu \mathrm{m}$

858

859

Figure S5 (continued). orco Receptor to Glomerulus 2D Map. 

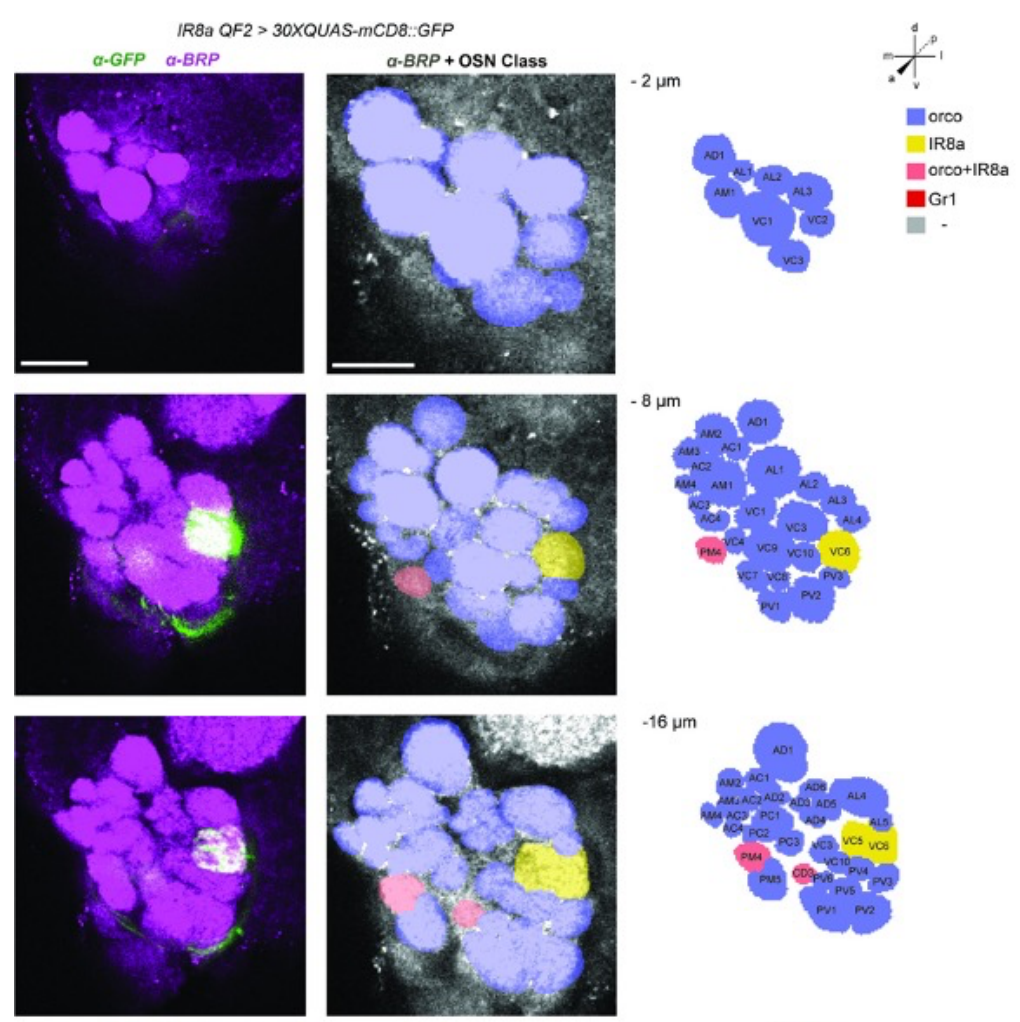

$-16 \mu \mathrm{m}$
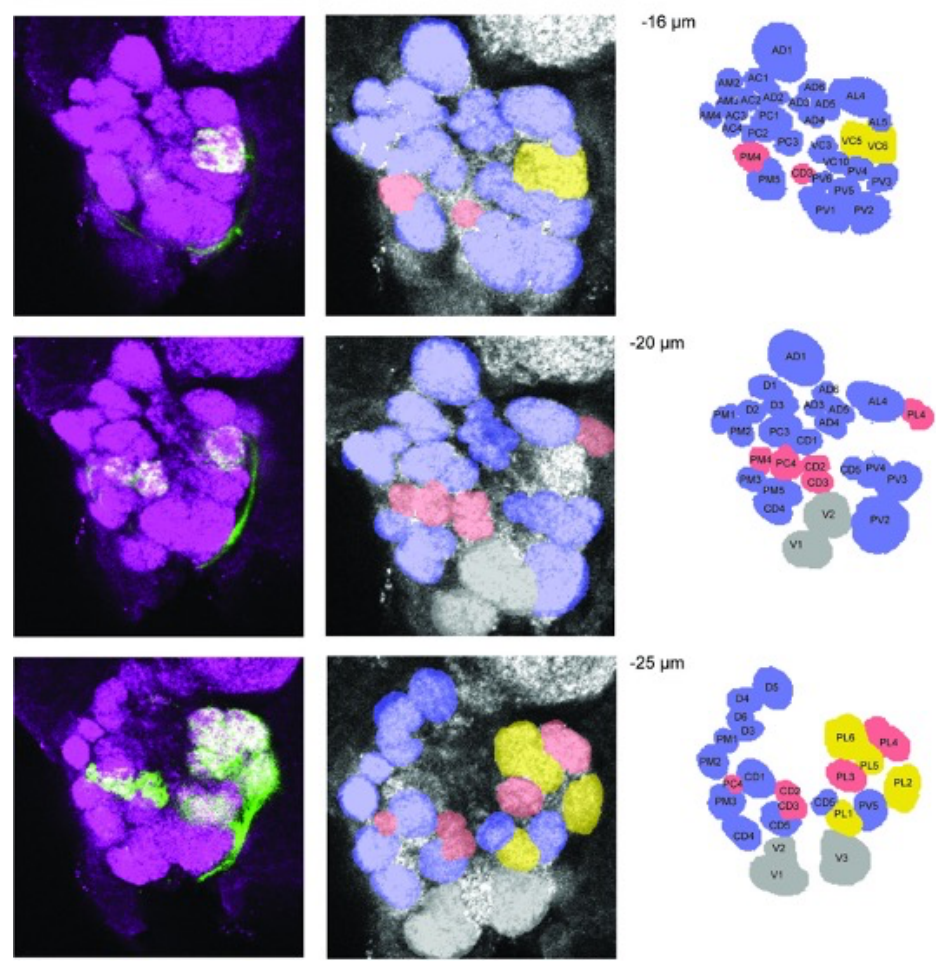

Figure S6. IR8a Receptor to Glomerulus 2D Map

864 Representative confocal stack from the left antennal lobe of an adult female Ir8a ${ }^{Q F 2}>30 X Q U A S-m C D 8:: G F P$ mosquito. 7 representative focal planes from a total of 44 z-stacks are shown on this map. Ir8a neurons innervate 15 total glomeruli: 2 glomeruli of the anteriorly positioned VC group (VC5 and VC6), 9 glomeruli of the PL group (PL1-9), 2 CD glomeruli (CD2 and CD3), 1 PC group glomerulus (PC4) and 1 PM group glomerulus (PM4). Glomeruli CD2, CD3, PL3, PL4, PC4, PM4 are putatively co-innervated by orco (+) and IR8a (+) neurons. IR8a (+) glomeruli are spatially invariant.

869 Scale bar: $10 \mu \mathrm{m}$. 
IR8a QF2 > 30XQUAS-mCD8::GFP
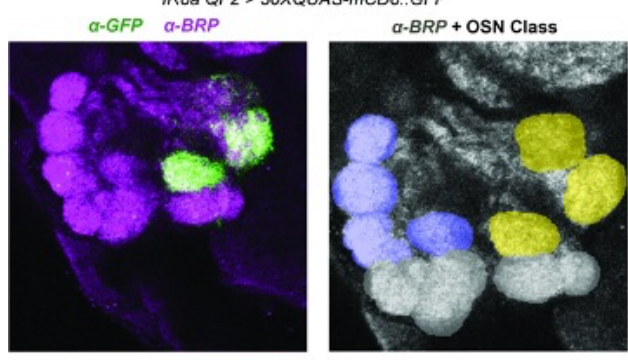

$-34 \mu \mathrm{m}$
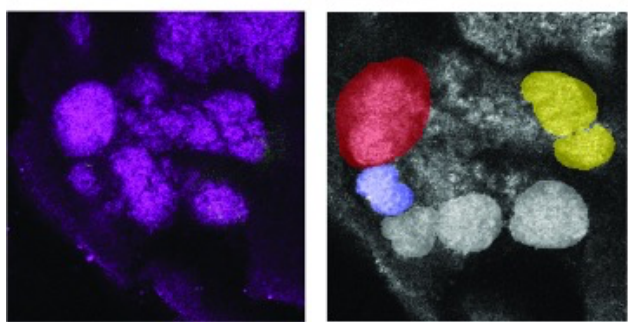

\section{$-39 \mu \mathrm{m}$}

Figure S6 (continued). IR8a Receptor to Glomerulus 2D Map.

876 

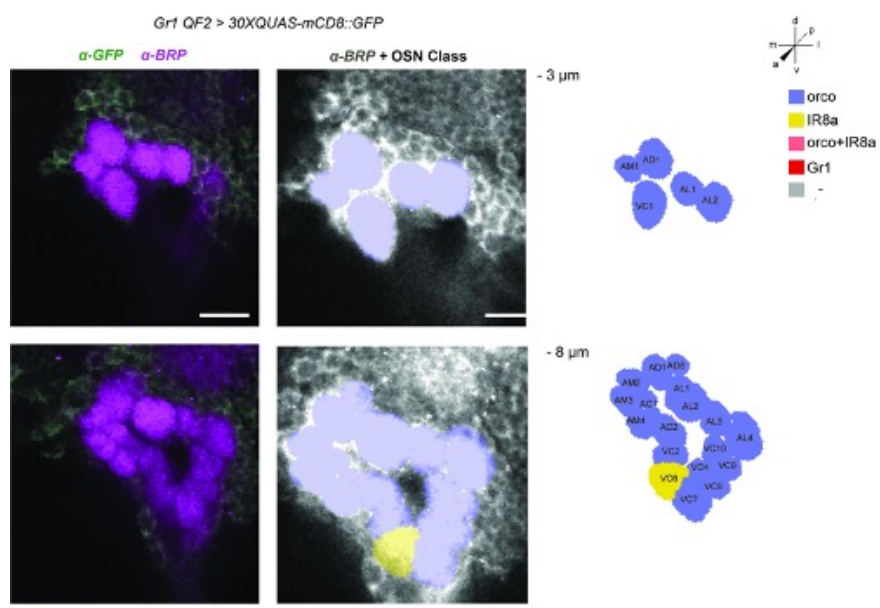

$-8 \mu \mathrm{m}$
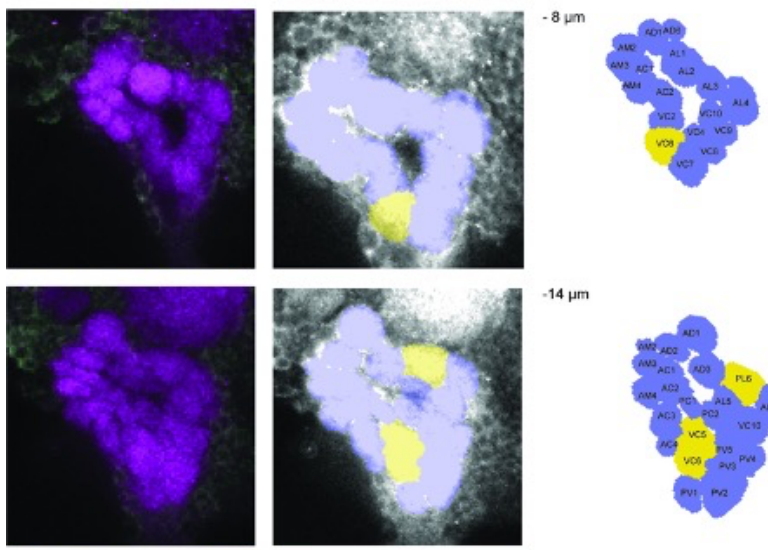

$-14 \mu \mathrm{m}$
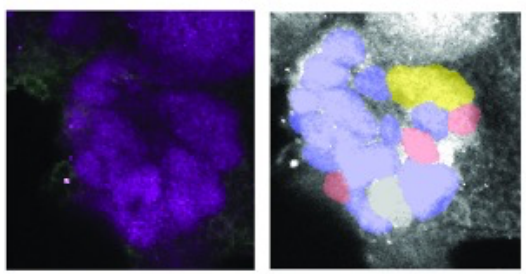

$-21 \mu \mathrm{m}$
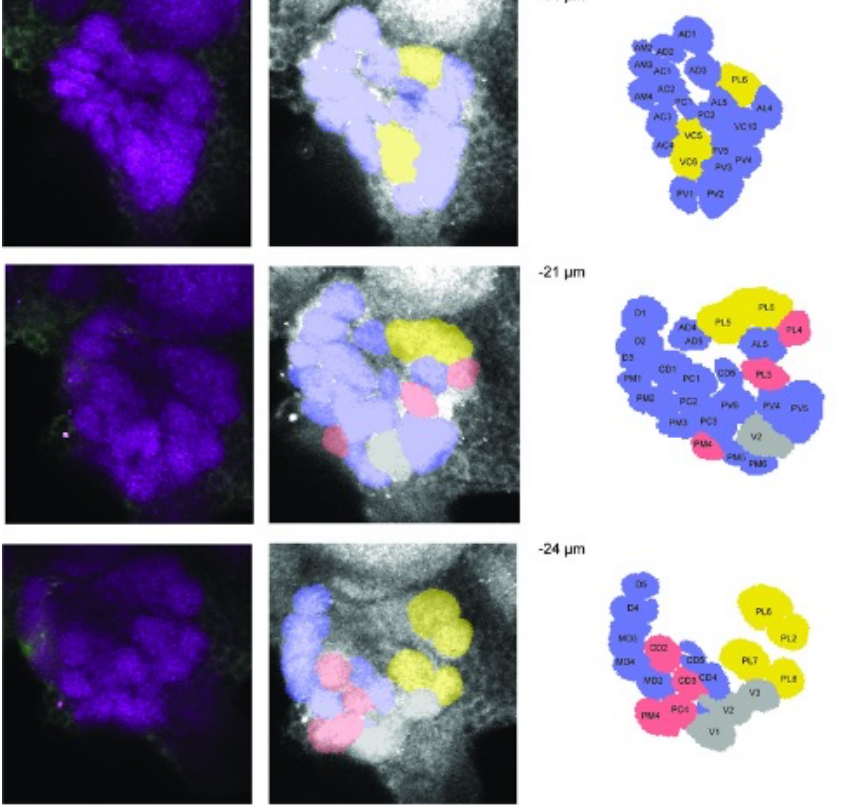

\section{Figure S7. Gr1 Receptor to Glomerulus 2D Map}

880 Representative confocal stack from the left antennal lobe of an adult female Gr1 ${ }^{\text {QF2 }}>30$ XQUAS-mCD8::GFP mosquito.

8817 representative focal planes from a total of $50 \mathrm{z}$-stacks are shown on this map. Scale bar: $10 \mu \mathrm{m}$. Gr1 neurons project

882 to the spatially invariant MD1 glomerulus. No innervation of Orco, Ir8a or GR1 neurons was observed in the Ventral (V)

883 group. Scale bar: $10 \mu \mathrm{m}$. 
bioRxiv preprint doi: https://doi.org/10.1101/2020.11.02.365916; this version posted November 4, 2020. The copyright holder for this preprint (which was not certified by peer review) is the author/funder. All rights reserved. No reuse allowed without permission.

Gr1 QF2 > 30XQUAS-mCD8::GFP

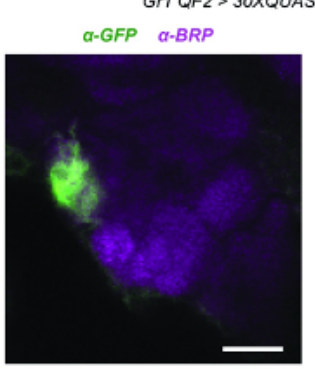

$a-B R P+$ OSN Class

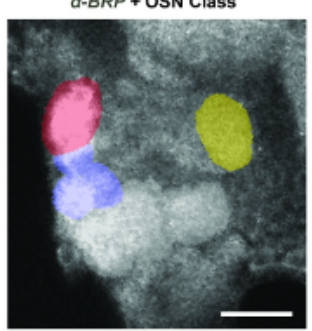

$-33 \mu \mathrm{m}$
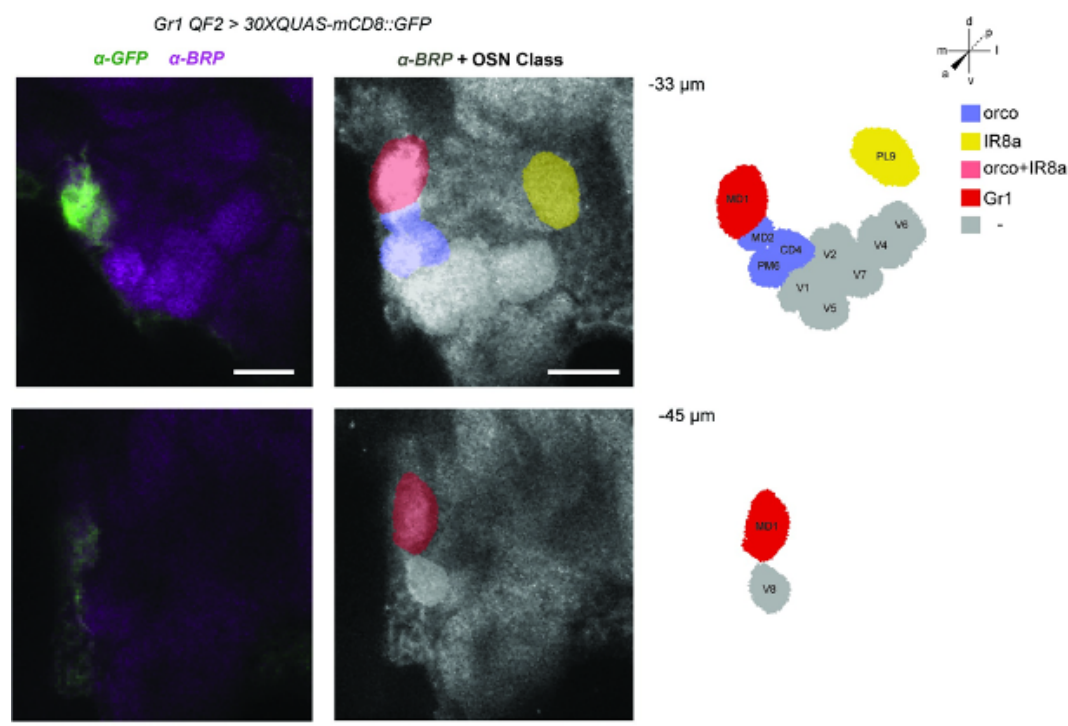

$-45 \mu \mathrm{m}$

$\square \mathrm{Gr} 1$

Figure S7 (continued). Gr1 Receptor to Glomerulus 2D Map. 
a<smiles>CC(C)(C)C(C)(C)C</smiles>

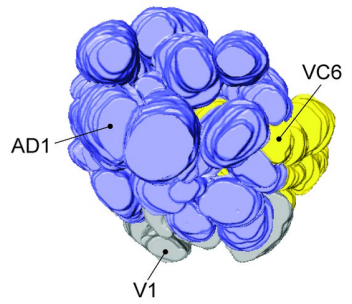

$10 \mu \mathrm{m}$

b $-9 \mu \mathrm{m}$

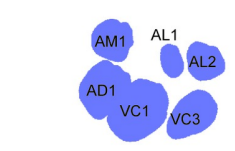

$-26 \mu \mathrm{m}$

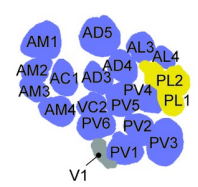

$-43 \mu \mathrm{m}$

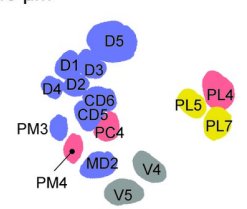

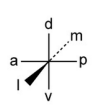

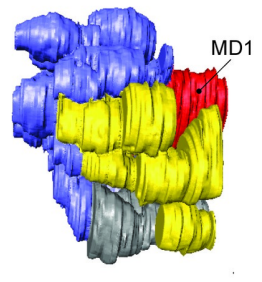

$\mu \mu \mathrm{m}$

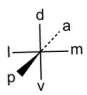

PL9

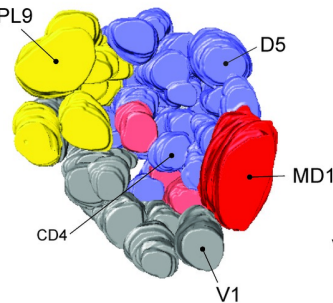

v1

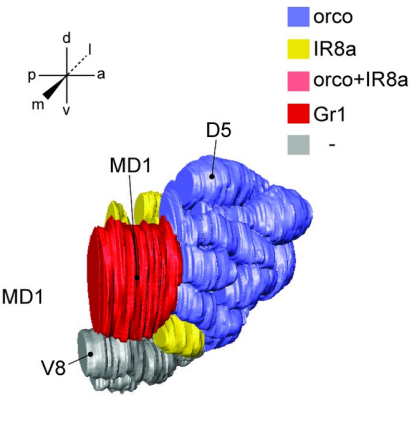

$-19 \mu \mathrm{m}$

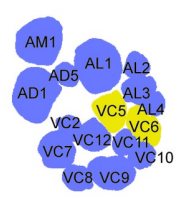

$-30 \mu \mathrm{m}$
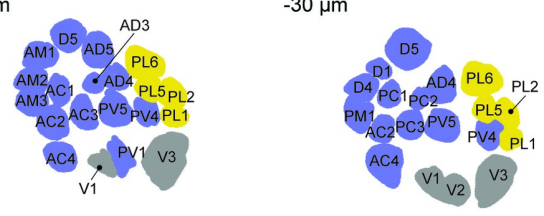

$-59 \mu \mathrm{m}$

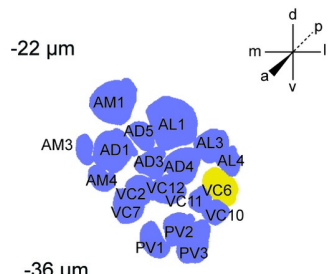

$-36 \mu \mathrm{m}$

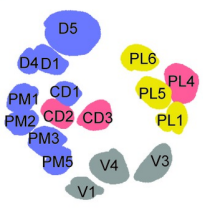

$-66 \mu \mathrm{m}$

Figure S8: Phalloidin-stained LVPib12 female antennal lobe model used as a reference map for CaMPARI2 activity dependent labeling.

(a) 3D model generated from a female LVib12 left antennal lobe stained with Alexa Fluor 647-phalloidin and imaged at 63X magnification from anterior, lateral, posterior and medial perspectives. Glomeruli are color coded according to chemoreceptor class. Landmark glomeruli are highlighted on the model. (b) 2D reference map for CaMPARI labelling. Scale bar: $10 \mu \mathrm{m}$. 
IR8a QF2 > 30XQUAS-CaMPARI2
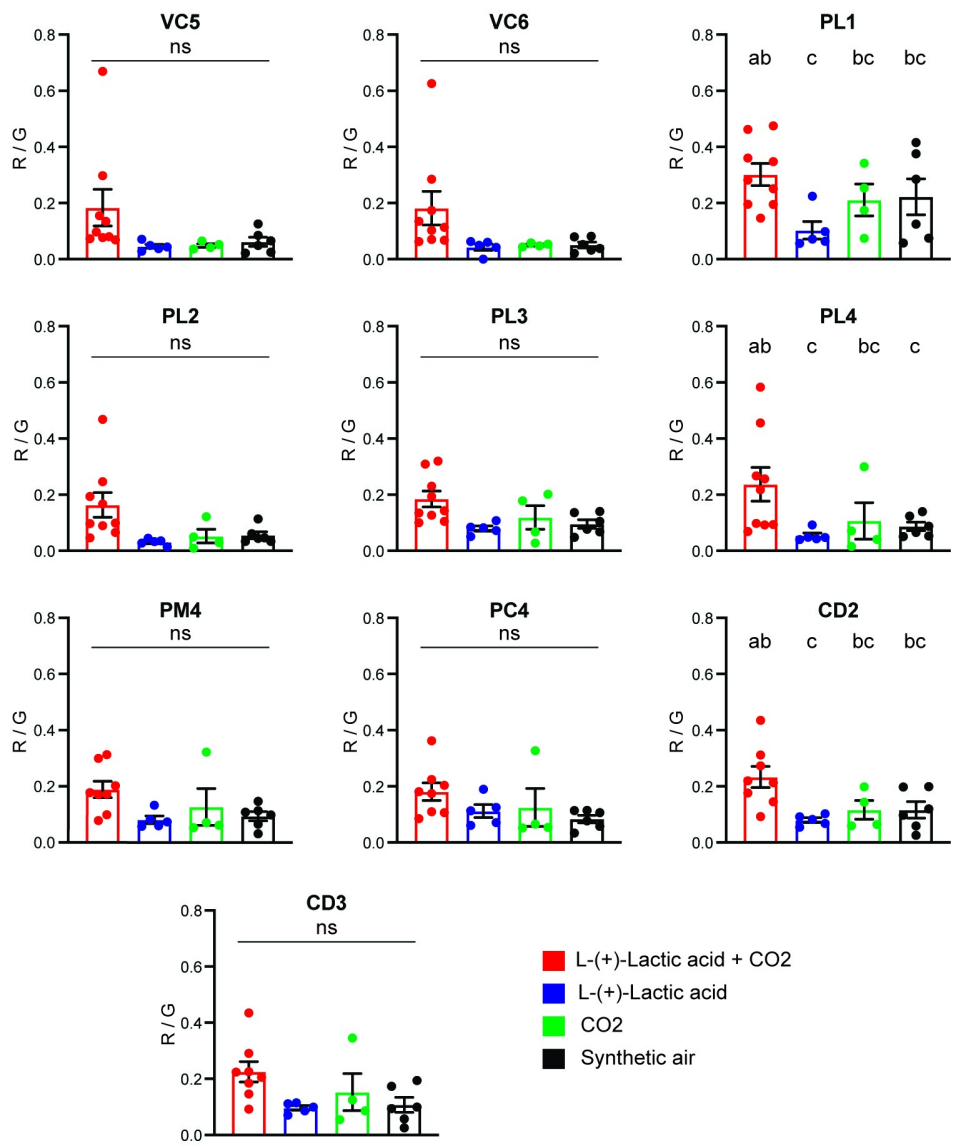

$$
\begin{aligned}
& \text { L-(+)-Lactic acid + CO2 } \\
& \text { L-(+)-Lactic acid } \\
& \text { CO2 } \\
& \text { Synthetic air }
\end{aligned}
$$

\section{Figure S9: CaMPARI2 Photoconversion In Supplemental IR8a (+) Glomeruli Screened in Response to Unitary} and Binary Blends of $\mathrm{L}-(+)$-lactic acid and $\mathrm{CO}_{2}$.

Comparisons of CaMPARI2 photoconversion revealed no significant differences in glomerular photoconversion in VC5, VC6, PL2, PL3, PM4 and CD3 glomeruli with any of the four odorant stimuli (L-(+)-lactic acid + CO2; L-(+)-lactic acid alone, CO2 alone; and synthetic air). Of the remaining glomeruli, PL5 and PL6 reported in the main text, and PL4 and CD2 glomeruli reported here exhibited photoconversion ratios where the ' $\mathrm{L}-(+)-$ lactic acid + CO2' stimulus had mean $\mathrm{R} / \mathrm{G}$ values that were significantly elevated relative to all or some of the other stimuli. For example, the PL4 glomerulus had mean photoconversion values for ' $L-(+)$-lactic acid $+\mathrm{CO} 2$ vs Synthetic Air', $P=0.0320$; and ' $L-(+)$-lactic acid + CO2 vs $L-(+)$-lactic acid', $P=0.0094$; that were significantly different. For the CD2 glomerulus, differences between mean $\mathrm{R} / \mathrm{G}$ values for 'L-(+)-lactic acid + CO2 vs $\mathrm{L}-(+)$-lactic acid' also reached statistical significance $(P=0.0481)$. Tukey's Multiple Comparison Test, $n=4-9$ brains per stimulus, mean R/G values +/- s.e.m. plotted. 

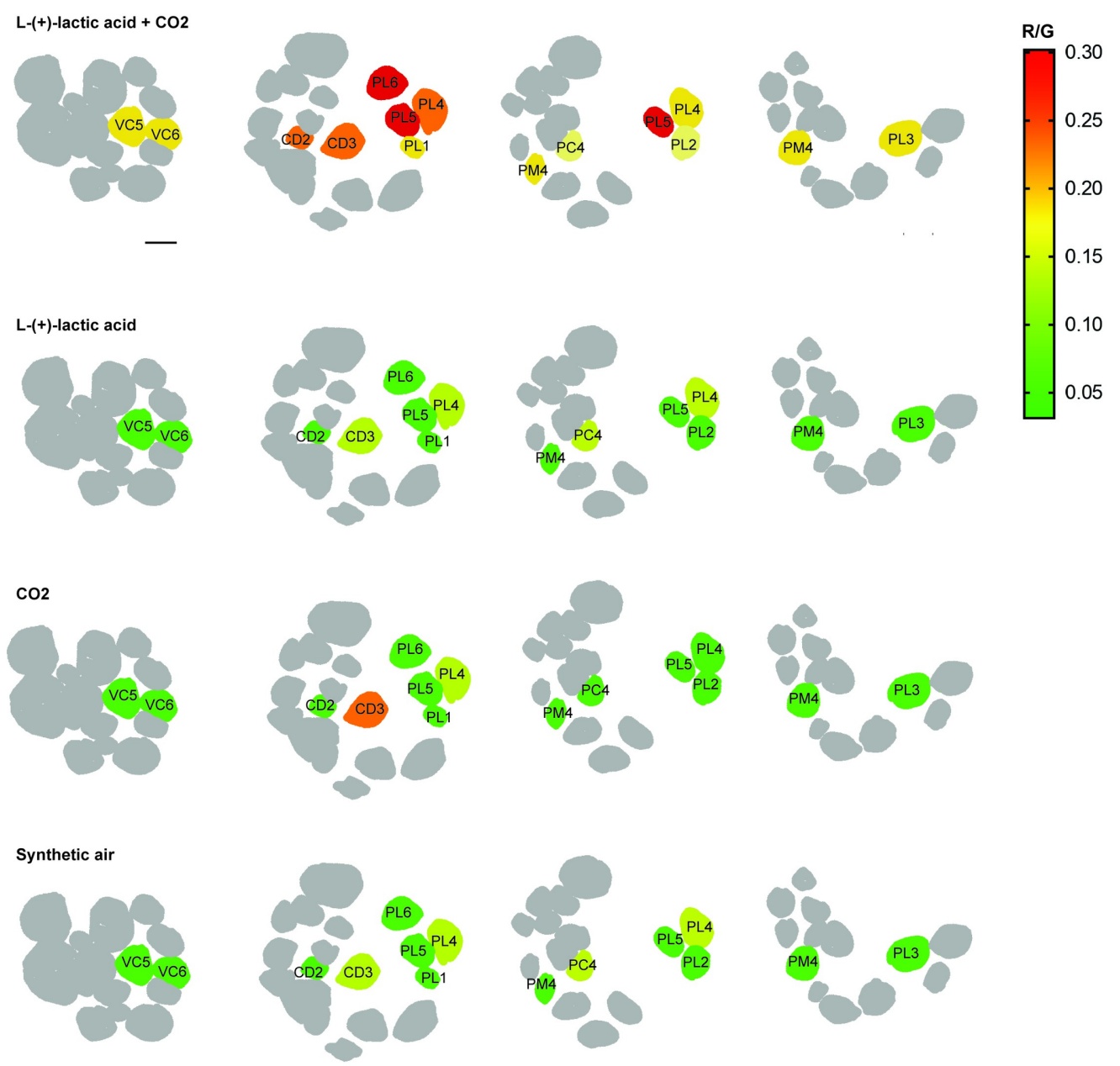

Figure S10: Mean Red/Green CaMPARI2 photoconversion values of IR8a (+) glomeruli plotted as a heat map on the phalloidin reference atlas. Average photoconversion $(R / G)$ values for $L(+)$-lactic acid $+1 \% \mathrm{CO}_{2} ; \mathrm{L}(+)$-lactic acid alone, $\mathrm{CO}_{2}$ alone; and a synthetic air control are shown. Scale bar: $10 \mu \mathrm{m}$. In total 12 out of 15 total IR8a (+) were included in this screen, with three IR8a (+) glomeruli (PL7, PL8 and PL9) not included because they were not clearly identified across all brain samples. 


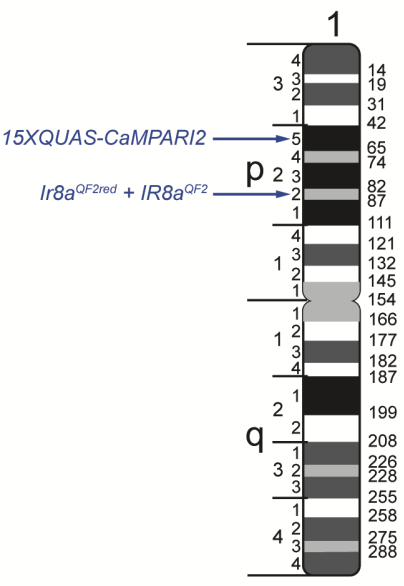

930 PCR.
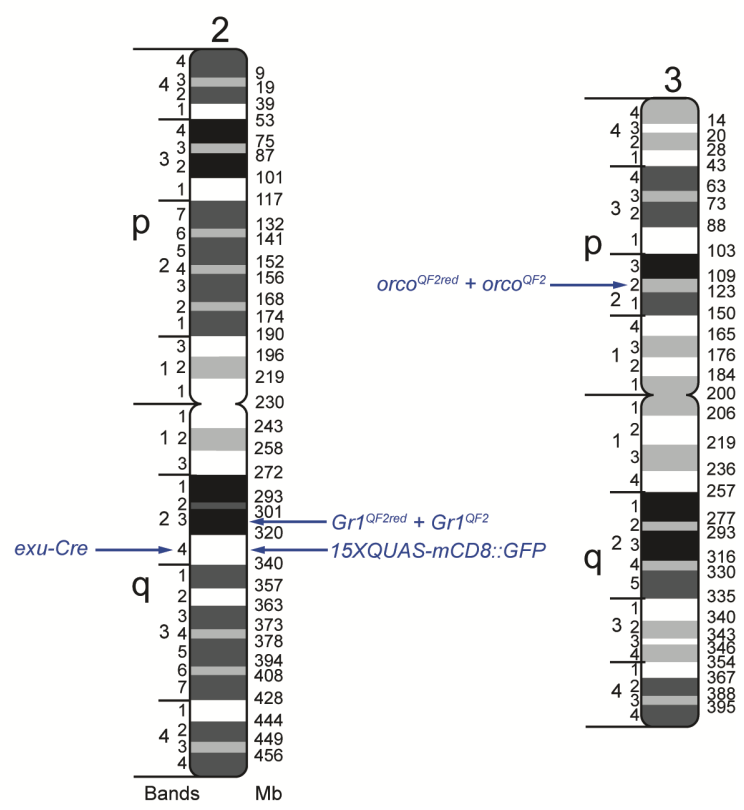

Figure S11. Physical Positions of Transgenes on Aedes aegypti Chromosome 1, 2 and 3. T2A-QF2 in-frame fusion cassettes were precisely inserted at orco (AAEL005776), IR8a (AAEL002922) and Gr1 (AAEL002380) loci via CRISPR-Cas9 homologous recombination, while exu-Cre, 15XQUAS-mCD8::GFP and 15XQUAS-CaMPARI2, were inserted into the mosquito genome using Mos1 mariner transposition with integration sites mapped using Splinkerette 
937 Table S1. CRISPR Target Sites and Locations

\begin{tabular}{|l|l|l|l|l|l|}
\hline Gene & Identifier & Chromosome & Band & CRISPR target site (with NGG) & Cut Site \\
\hline orco & AAEL005776 & 3 & $3 \mathrm{p} 22$ & CCATCAAGGCTTGGTACCCG TGG & Exon 3 \\
\hline IR8a & AAEL002922 & 1 & $1 \mathrm{p} 22$ & TCCAACGCGAAAGTACCGCT TGG & Exon 3 \\
\hline Gr1 & AAEL002380 & 2 & $2 \mathrm{q} 23$ & TGACCATGAGGTACTTATAC TGG & Exon 3 \\
\hline
\end{tabular}

938

939

Table S2. Homology Arms for T2A-QF2 Donor Constructs

940

\begin{tabular}{|l|l|l|}
\hline Gene region & $\begin{array}{l}\text { Homology Arm Size } \\
(\mathbf{b p})\end{array}$ & Homology Arm Primers with In-Fusion Adapters (underlined) \\
\hline Gr1 Left Arm & 1003 & $\begin{array}{l}\text { 5'-TATAACCCGCCTCGGTTATTCCGTTTGTTTTCAATTTTCCGCG-3' } \\
\text { 5'-TGCCGCGGCCCTCTCCGCTTCCCTGGTAGTCGGTCCACATGT-3' }\end{array}$ \\
\hline Gr1 Right Arm & 884 & $\begin{array}{l}\text { 5'-TGACAGATCTGCGCGTAAGTACCTCATGGTCACCGGA-3' } \\
\text { 5'-GATATCGATCGCGCGACCGTTTGGAGGTTGAATTG-3' }\end{array}$ \\
\hline Orco Left Arm & 2624 & $\begin{array}{l}\text { 5'-TATAACCCGCCTCGGTGCAAGTGGATCATTTGTCG-3' } \\
\text { 5'-TGCCGCGGCCCTCTCCGCTTCCGTACCAAGCCTTGATGGGC-3' }\end{array}$ \\
\hline Orco Right Arm & 1329 & $\begin{array}{l}\text { 5'-TGACAGATCTGCGCGCCGTGGGATGCAATGAG-3' } \\
\text { 5'-GATATCGATCGCGCGGTGCAATTGTGCCATTTTGA-3' }\end{array}$ \\
\hline IR8a Left Arm & 2159 & $\begin{array}{l}\text { 5'-TATAACCCGCCTCGGCAAAGTATAATTTCGCCCCCTCC-3' } \\
\text { 5'-TGCCGCGGCCCTCTCCGCTTCCCTTGGTCGGTTTGATCTTCTG-3' }\end{array}$ \\
\hline IR8a Right Arm & 1964 & $\begin{array}{l}\text { 5'-TGACAGATCTGCGCGGGTACTTTCGCGTTGGAACTA-3' } \\
\text { 5'-GATATCGATCGCGCGCTCTATGGCAGCCAAGATATTGG-3' }\end{array}$ \\
\hline
\end{tabular}

941

942 


\section{Table S3. Template Materials for Constructs}

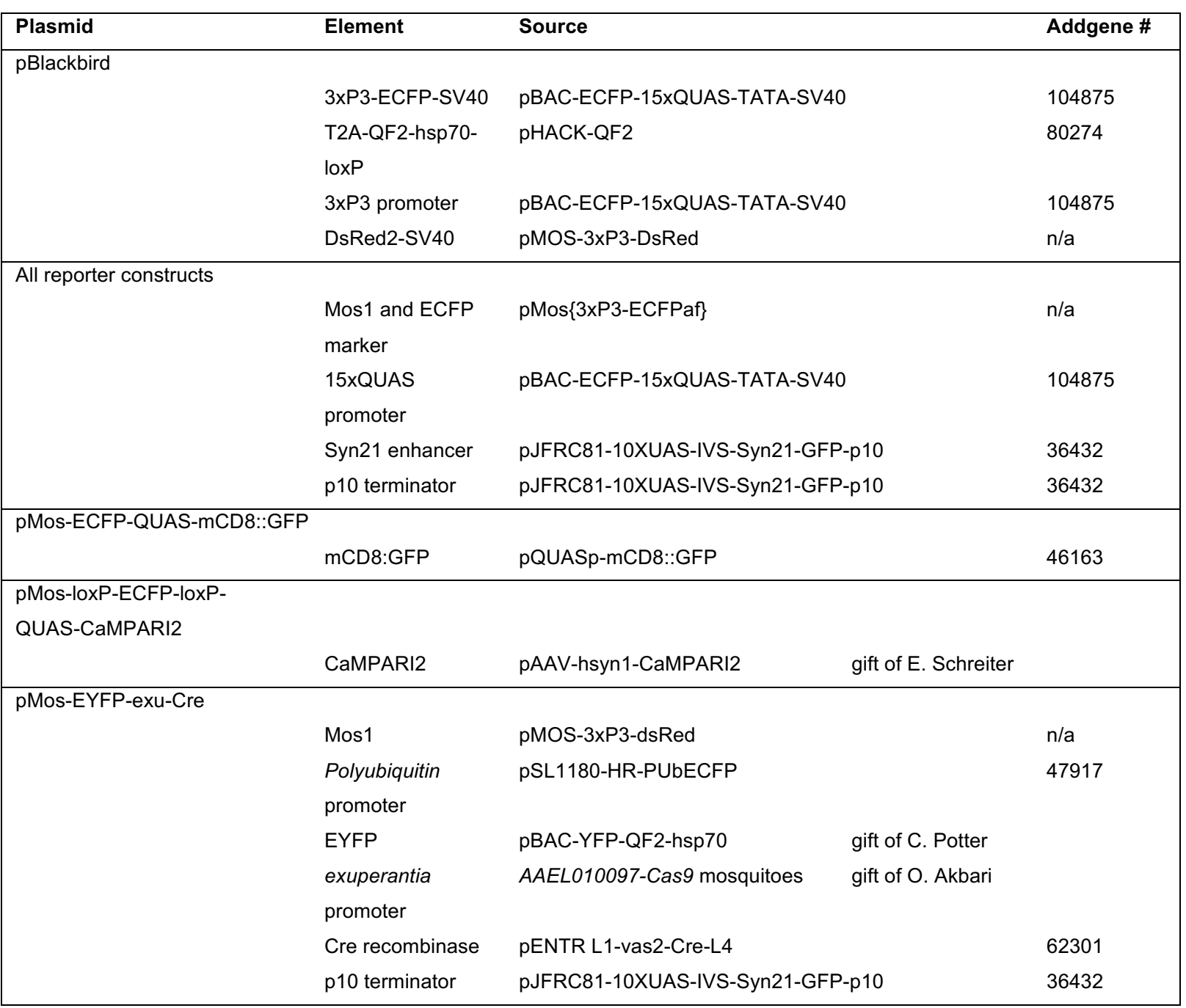


Table S4. Complete Genotypes of Aedes aegypti Stocks and Composite Genotypes

947

\begin{tabular}{|c|c|c|}
\hline Identifier & ABBREVIATED GENOTYPE & $\begin{array}{l}\text { FULL GENOTYPE (Aedes aegypti chromosome 1; 2; 3) } \\
+=\text { wild-type allele }\end{array}$ \\
\hline Stock & LVPib12 & $+/+;+/+;+/+$ \\
\hline Stock & $\begin{array}{l}\text { marker }=3 \times P 3-d s R e d 2, \\
3 \times P 3-E C F P\end{array}$ & $+/+;$ Gr1 ${ }^{\text {QF2Red }} /$ Gr1 $1^{\text {QF2Red }} ;+/+$ \\
\hline Stock & marker $=3 \times P 3-d s R e d 2$ & IR8a $a^{Q F 2 R e d} / / I R 8 a^{Q F 2 R e d} ;+/+;+/+$ \\
\hline Stock & marker $=3 \times P 3-d s R e d 2$ & $+/+;+/+;$ orco $^{Q F 2 R e d} /+$ \\
\hline Stock & marker-free & $+/+; G r 1^{Q F 2} / G r 1^{Q F 2} ;+/+$ \\
\hline Stock & marker-free & $I R 8 a^{Q F 2} / / R 8 a^{Q F 2} ;+/+;+/+$ \\
\hline Stock & marker-free & $+/+;+/+; \operatorname{orco}^{Q F 2} /+$ \\
\hline Stock & marker $=3 \times P 3-E C F P$ & +/+; 15XQUAS-mCD8::GFP / 15XQUAS-mCD8::GFP; +/+ \\
\hline Stock & $\begin{array}{l}\text { 15XQUAS- } \quad \text { marker }=3 \times P 3-E C F P \\
\text { CaMPARI2 }\end{array}$ & 15XQUAS-CaMPARI2 / 15XQUAS-CaMPARI2; +/+; +/+ \\
\hline Stock & marker $=P U b-E Y F P$ & $+/+;$ exu-Cre / +; +/+ \\
\hline Composite & $G r 1^{Q F 2}>30 X Q U A S-m C D 8:: G F P$ & $\begin{array}{l}+/+; 15 X Q U A S-m C D 8:: G F P, \text { Gr1 }{ }^{\text {QF2 }} / 15 X Q U A S-m C D 8:: G F P \\
+/+\end{array}$ \\
\hline Composite & $\operatorname{orco}^{Q F 2}>30 X Q U A S-m C D 8:: G F P$ & +/+; 15XQUAS-mCD8::GFP / 15XQUAS-mCD8::GFP; orco ${ }^{Q F 2} /+$ \\
\hline Composite & $I R 8 a^{Q F 2}>30 X Q U A S-m C D 8:: G F P$ & IR8a ${ }^{Q F 2} /+;$ 15XQUAS-mCD8::GFP / 15XQUAS-mCD8::GFP; +/+ \\
\hline Composite & Gr1 ${ }^{\text {QF2Red }}>15 X Q U A S-m C D 8:: G F P$ & +/+; Gr1 ${ }^{\text {QF2Red } / 15 X Q U A S-m C D 8:: G F P ; ~+/+~}$ \\
\hline Composite & orco $^{Q F 2 R e d}>15 X Q U A S-m C D 8:: G F P$ & +/+; 15XQUAS-mCD8::GFP / +; orco oF2Red $/+^{\text {+ }}$ \\
\hline Composite & IR8a $a^{\text {QF2Red }}>15 X Q U A S-m C D 8:: G F P$ & IR8a $a^{\text {QF2Red } /+; ~ 15 X Q U A S-m C D 8:: G F P ~ / ~+; ~+/+~}$ \\
\hline Composite & $I R 8 a^{Q F 2}>30 X Q U A S-C a M P A R / 2$ & IR8a ${ }^{Q F 2}, 15 X Q U A S-C a M P A R I 2 / 15 X Q U A S-C a M P A R I 2 ;+/+;+/+$ \\
\hline Composite & $\mathrm{Gr1}^{\mathrm{QF2}}>30 \mathrm{XQUAS-CaMPAR/2}$ & 15XQUAS-CaMPARI2 / 15XQUAS-CaMPAR/2; Gr1 ${ }^{\text {QF2 } /+;+/+}$ \\
\hline Composite & orco $^{Q F 2}>15 X Q U A S-C a M P A R / 2$ & 15XQUAS-CaMPARI2 / +; +/+; orco ${ }^{Q F 2} /+$ \\
\hline
\end{tabular}

948 
bioRxiv preprint doi: https://doi.org/10.1101/2020.11.02.365916; this version posted November 4, 2020. The copyright holder for this preprint (which was not certified by peer review) is the author/funder. All rights reserved. No reuse allowed without permission.

950 Table S5. Genomic Integration Sites of Mos1 mariner transgenes

\begin{tabular}{|l|l|l|l|l|}
\hline Transgene & Chromosome & Band & Insertion Site & Insertion Location \\
\hline 15XQUAS-CaMPAR/2 & 1 & $1 \mathrm{p} 25$ & 65092968 & AAEL026960, Intron \\
\hline 15XQUAS-mCD8::GFP & 2 & $2 \mathrm{q} 24$ & 329084910 & $\begin{array}{l}\text { Upstream of AAEL021072 } \\
\text { IncRNA }\end{array}$ \\
\hline exu-Cre & & & & AAEL013635, Intron \\
\hline
\end{tabular}

951

952

953

954

955

956 